\title{
Making the most of fleets: A profit- maximizing multi-vehicle pickup and delivery selection problem
}

\section{Journal Article}

\section{Author(s):}

Qiu, Xiaoqiu; Feuerriegel, Stefan; Neumann, Dirk

Publication date:

2017-05-16

Permanent link:

https://doi.org/10.3929/ethz-b-000182391

\section{Rights / license:}

In Copyright - Non-Commercial Use Permitted

Originally published in:

European Journal of Operational Research 259(1), https://doi.org/10.1016/j.ejor.2016.10.010 


\title{
Making the most of fleets: A profit-maximizing multi-vehicle pickup and delivery selection problem
}

\author{
Xiaoqiu Qiu ${ }^{\mathrm{a}}$, Stefan Feuerriegel ${ }^{\mathrm{a}, *}$, Dirk Neumann ${ }^{\mathrm{a}}$ \\ ${ }^{a}$ Chair for Information Systems Research, University of Freiburg, Platz der Alten Synagoge, 79098 \\ Freiburg, Germany
}

\begin{abstract}
Road freight transportation is a pillar of the modern economy. Despite the increasing competition, over $20 \%$ of all vehicles run empty on European roads. As a remedy, freight exchanges have been established to bridge such supply-demand imbalances, with the largest markets trading over 200,000 daily offers. Carriers searching for profit-maximizing freights on such markets face a Profit-Maximizing Pickup and Delivery Selection Problem (PPDSP) that has not yet been addressed in previous research. In this paper, we develop a novel graph search that branches on feasible routes for an exact solution and, based on this, we develop a randomized search heuristic for the single vehicle case, a greedy heuristic for the multi-vehicle case, and a Maximum Set Packing formulation for the case of homogeneous and heterogeneous fleets. Computational experiments show that most instances of the various setups can be solved optimally and much faster than the solution offered by the Gurobi optimizer. Both heuristics are highly efficient and the problem of fleets can be solved almost as quickly as the single vehicle case.
\end{abstract}

Keywords: Decision Support Systems, Transportation, Logistics, Branch and bound, Heuristics

\section{Introduction}

Road freight transportation has become an integral element of the globalized world: more cargo is shipped on roads than rail, water and air shipment combined. Yet over $20 \%$ of all vehicles are traveling empty. ${ }^{1}$ This creates unnecessary carbon

\footnotetext{
*Corresponding author. Mail: stefan.feuerriegel@is.uni-freiburg.de; Tel: +49761203 2395; Fax: +497612032416.

Email addresses: jimworks@gmail .com (Xiaoqiu Qiu), stefan.feuerriegel@is.uni-freiburg.de (Stefan Feuerriegel), dirk.neumann@is.uni-freiburg.de (Dirk Neumann)

${ }^{1}$ Eurostat: European statistics transportation database. Statistics data. URL: http://epp. eurostat.ec.europa.eu/portal/page/portal/transport/data/database. Accessed on
} 
emissions footprints by wasting huge residual capacities. Instead, excess capacity can be traded by logistics carriers on freight exchanges as a remedy. As such, freight exchanges represent a lever to address the increasing competition in almost every industry, especially in the transportation sector, as well as the demand for more agile and adaptable supply chains (Lee, 2004).

The concept of freight exchanges is not now; there are over 100 exchanges online in Europe, ranging from simple bulletin boards to international exchanges that trade 1.2 million tons of freight daily. ${ }^{2}$ Carriers trading on such markets face a new challenge: identifying the most profitable route from among the 200,000 daily offers.

Traditional operations research has addressed this problem for truckload carriers, which ship only one request at a time. This can be modeled as a mixed graph with requests as directed arcs and inter-request routes as undirected edges. The aim is to find the minimal cost route that traverses all arcs as in the Stacker Crane Problem (Coja-Oghlan et al., 2006), or the most profitable route that traverses a selective subset of the arcs, i. e. the Orienteering Problem (Vansteenwegen et al., 2011). Truckload problems are relatively simple since each delivery must be made right after its pickup and, hence, each request can be treated as a node, making the problem similar to the classic Vehicle Routing Problem (Toth \& Vigo, 2002).

In contrast, the less-than-truckload (LTL) version has been considered only rarely. LTL carriers consolidate multiple requests in one vehicle and transport them together, as into parcel delivery, for example. Consequently, they must consider not only request-specific attributes, but also whether multiple requests fit together into the delivery plan. The cost minimizing case is often modeled as the Pickup and Delivery Problem (PDP), while the profit maximizing case with selective pickup has not been studied thus far. Solving this problem optimally and quickly helps to improve the operational efficiency of the carriers, and contributes to greener transportation.

In this paper, we propose a novel Profit-Maximizing Pickup and Delivery Selection Problem (PPDSP) with time windows and selective pickups. The contributions are threefold:

1. We develop a simple graph search that enumerates feasible routes under pickup and delivery constraints, which solves the multi-vehicle PPDSP optimally and reaches the solution much faster than the state-of-the-art Gurobi optimizer (Gurobi, 2014).

2. We develop two heuristics: (i) a randomized search for the single vehicle case and (ii) a greedy heuristic for the multi-vehicle case. Both of these are fast and highly efficient.

3. For the case of fleets, we show that the problem can be modeled as a Maximum

June 10, 2014.

${ }^{2}$ Teleroute: Teleroute freight exchange. URL: http://www. teleroute.com/. Accessed on June 10, 2014. 
Set Packing problem upon the feasible routes, and that an optimal solution may be found to optimum in approximately the same time as in the single vehicle case.

The remainder of the paper is structured as follows: Section 2 surveys related works on the Pickup and Delivery Problem, as well as routing problems with selective pickup. Section 3 defines the model and its assumptions, while Section 4 presents the optimization algorithms. This is followed by computational experiments in Section 5 . Section 6 concludes and presents an outlook for future research.

\section{Related work}

Our Profit-Maximizing Pickup and Delivery Selection Problem (PPDSP) is a transportation optimization problem characterized by three aspects: (i) profit-maximizing routing, (ii) pickup and delivery and (iii) selective pickup. Our following literature review shows that each of these aspects has been studied on its own but that their combination has been neglected in prior research. Altogether, the corresponding classification is illustrated in Figure 1.

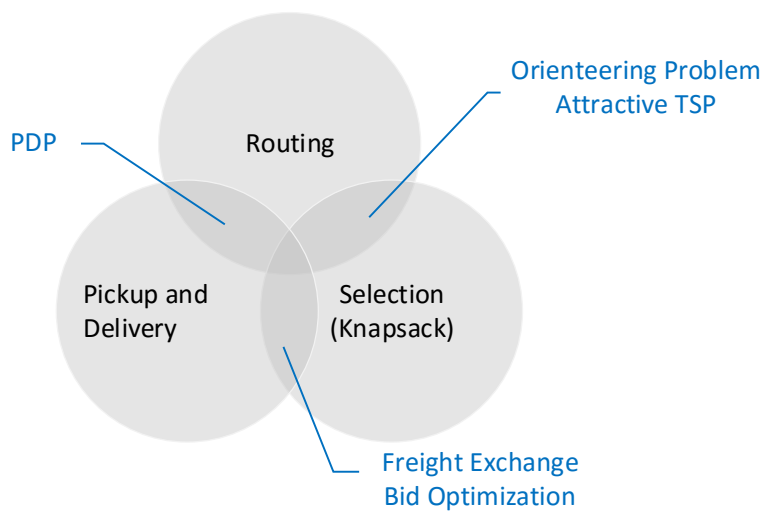

Figure 1: Classification of related problems with the Profit-Maximizing Pickup and Delivery Selection Problem located in the central intersection.

Each of the three previously mentioned problem characteristics has been covered extensively in operations research (Toth \& Vigo, 2002): routing is solved in the naïve Traveling Salesman Problem (TSP) and Vehicle Routing Problem (VRP). These are then augmented with request pickups and deliveries in the Pickup and Delivery Problem (PDP). The PDP is a large family of vehicle routing problems that transport objects or people between origins and destinations (Berbeglia et al., 2007). Most PDPs aim to find the route with minimal cost under side constraints. A comprehensive survey of PDPs can be found in Parragh et al. (2008). 
PDPs are frequently classified into three groups according to the number of origins and destinations (Berbeglia et al., 2007) - many-to-many, one-to-many-to-one and one-to-one - as follows (see Table 1): the many-to-many case considers only one or a few objects, which can be transported between any locations. Examples are refueling petrol stations or redistributing rental bikes (Ting \& Liao, 2013). The exact solutions usually utilize branch-and-cut approaches (Hernández-Pérez \& SalazarGonzález, 2007, 2014) and necessitate (meta-)heuristics for large instances (Mladenović et al., 2012). The one-to-many-to-one case introduces two depots; then, objects are transported from the depot to customers in order to satisfy demand or from the customers back to the depot. Examples include supplying beverages to supermarkets and collecting the empty bottles (Gribkovskaia et al., 2008). Here, Gutiérrez-Jarpa et al. (2010) investigate a variant with selective pickups, while Masson et al. (2014) study an application to shuttle routes. Our PPDSP falls into the one-to-one case where each object or request is unique and associated with one origin and a corresponding destination.

Several approaches to one-to-one PDPs are known. As this problem does not allow for trans-shipment, it often occurs as a Pickup and Delivery Traveling Salesman Problem (Dumitrescu et al., 2010; Hernández-Pérez \& Salazar-González, 2009; RodríguezMartín \& Salazar-González, 2011), or PDP with time windows (Ropke \& Cordeau, 2009; Cherkesly et al., 2015). The largest problem size that can currently be solved optimally contains around 500 requests with tight time windows using a branch-cutprice algorithm. A highly effective variant of the latter algorithm has been proposed, for instance, in Baldacci et al. (2011). When transporting people, e. g. when routing ambulances, the one-to-one PDP is referred to as the Dial-a-Ride Problem, DARP for short (Cordeau \& Laporte, 2007). The instances which are solved optimally are usually relatively small, with the largest containing 58 requests (Parragh et al., 2012).

The General Vehicle Routing Problem (GVRP) resembles our definition, but without a total trip time (Goel \& Gruhn, 2008). In addition, it generalizes our problem by allowing each request to entail a sequence of delivery locations, instead of having only a single delivery location. Therefore, column generation is a viable approach to ordering the multiple tasks associated with each request (Goel, 2010). Furthermore, the GVRP is approached by an improvement heuristic for reduced neighborhoods, which optimizes a tour using operations, such as insertions, swaps and relocations (Goel \& Gruhn, 2008). However, the operators harness the fact that a request entails more than one delivery location, while being inept with only one drop-off. Accordingly, our work is closely linked to viable starting heuristics of the GVRP.

Table 1 summarizes recent studies on the Pickup and Delivery Problem. The problem size is measured by the number of objects or requests. Since one object is here associated with one pickup node and one delivery node, we report figures for which we divided the number of nodes by two for reasons of consistency.

The third characteristic of our problem, i. e. selective pickup, resembles the Knap- 


\begin{tabular}{|c|c|c|c|c|c|c|c|c|c|c|}
\hline \multirow[t]{2}{*}{ Model } & \multicolumn{3}{|c|}{ Node } & \multicolumn{2}{|c|}{ Vehicle } & \multicolumn{2}{|c|}{ Problem } & \multirow{2}{*}{$\begin{array}{c}\text { Variant } \\
\text { Opt. }\end{array}$} & \multirow[t]{2}{*}{ Solution } & \\
\hline & Sel. & Het. & TW & Cap. & Num. & Size & Max. $\mathrm{T}$ & & & \\
\hline \multirow[t]{4}{*}{ M-M } & & & & $\checkmark$ & 1 & 100 & & $\checkmark$ & 1-PDTSP & Hernández-Pérez \& Salazar-González (2007): branch-cut \\
\hline & & & & $\checkmark$ & 1 & 500 & & & 1-PDTSP & Mladenović et al. (2012): general variable neighborhood search \\
\hline & & $\checkmark$ & & $\checkmark$ & 1 & 30 & & $\checkmark$ & m-PDTSP & Hernández-Pérez \& Salazar-González (2014): branch-cut \\
\hline & $\checkmark$ & & & $\checkmark$ & 1 & 227 & & & SPDP & Ting \& Liao (2013): memetic algorithm; with 454 nodes \\
\hline \multirow{3}{*}{ 1-M-1 } & & $\checkmark$ & $\checkmark$ & $\checkmark$ & $m$ & 87 & & $\checkmark$ & PDPS & Masson et al. (2014): branch-cut-price \\
\hline & & $\checkmark$ & $\checkmark$ & $x$ & $m$ & 193 & & $\checkmark$ & PDPS & Masson et al. (2014): branch-cut-price \\
\hline & $\checkmark$ & $\checkmark$ & $\checkmark$ & $\checkmark$ & $m$ & 100 & & $\checkmark$ & VRPDSPTW & Gutiérrez-Jarpa et al. (2010): branch-price \\
\hline \multirow[t]{7}{*}{$1-1$} & & $\checkmark$ & & & 1 & 35 & & $\checkmark$ & TSPPD & Dumitrescu et al. (2010): branch-and-cut \\
\hline & & $\checkmark$ & & $\checkmark$ & 1 & 15 & & $\checkmark$ & m-PDTSP & Hernández-Pérez \& Salazar-González (2009): branch-cut \\
\hline & & $\checkmark$ & & $\checkmark$ & 1 & 15 & & & m-PDTSP & Rodríguez-Martín \& Salazar-González (2011): GRASP \\
\hline & & $\checkmark$ & & 1 & 1 & 100 & & & 1-DPDP & $\begin{array}{l}\text { Treleaven et al. (2013): SPLICE polynomial time alg.; with unit } \\
\text { capacity) }\end{array}$ \\
\hline & & $\checkmark$ & & $\checkmark$ & $m$ & 50 & & $\checkmark$ & PDP & Venkateshan \& Mathur (2011): column generation, branch-bound \\
\hline & & $\checkmark$ & $\checkmark$ & $\checkmark$ & $m$ & 75 & & $\checkmark$ & PDPTW-LIFO & Cherkesly et al. (2015): hybrid branch-cut-price \\
\hline & & $\checkmark$ & $\checkmark$ & $\checkmark$ & $m$ & 500 & & $\checkmark$ & PDPTW & Ropke \& Cordeau (2009): branch-cut-price; with tight TW \\
\hline \multirow[t]{2}{*}{ DARP } & & $\checkmark$ & $\checkmark$ & $\checkmark$ & 1 & 20 & $\checkmark$ & $\checkmark$ & DARP & $\begin{array}{l}\text { Häme (2011): adaptive insertion; with heuristic for up to } 50 \text { cus- } \\
\text { tomers }\end{array}$ \\
\hline & & $\checkmark$ & $\checkmark$ & $\checkmark$ & $m$ & 40 & $\checkmark$ & $\checkmark$ & DARP & $\begin{array}{l}\text { Parragh et al. (2012): column generation, variable neighborhood } \\
\text { search for up to } 273 \text { requests, } 30 \text { drivers and } 2 \text { types of vehicles }\end{array}$ \\
\hline \multirow[t]{2}{*}{ GVRP } & $\checkmark$ & $\checkmark$ & $\checkmark$ & $\sqrt{2}$ & $m$ & $100 /$ iter & & $\checkmark$ & GVRP & Goel \& Gruhn (2008): improvement heuristic for vehicle tours \\
\hline & $\checkmark$ & $\checkmark$ & $\checkmark$ & $\checkmark$ & $m$ & $100 /$ iter & & $\checkmark$ & GVRP & Goel (2010): column generation for vehicle tours \\
\hline PPDSP & $\checkmark$ & $\checkmark$ & $\checkmark$ & $\checkmark$ & $m$ & 500 & $\checkmark$ & $\checkmark$ & PPDSP & $\begin{array}{l}\text { This paper: graph search, randomized search, greedy search, } \\
\text { max. set packing for homogeneous fleets, all with profit maxi- } \\
\text { mization }\end{array}$ \\
\hline
\end{tabular}

Table 1: Recent studies on the Pickup and Delivery Problem and its variants.

sack problem in which the goal is to choose a profit-maximizing combination of requests that fits the vehicle's capacity and fulfills the delivery plan (e.g. constraints of time windows). Combined with routing, selective pickup appears as the Orienteering Problem (OP), whose aim is to find a route that visits as many "checkpoints" as possible in order to maximize rewards (Vansteenwegen et al., 2011). A similar problem is given by the Attractive Traveling Salesman Problem. The latter can, for instance, be optimally solved for instances of up to 400 vertices using branch-and-cut (Erdoğan et al., 2010).

In practice, carriers face a bid optimization on freight markets, which can be approach by finding an optimal combination of selective pickup and transportation requests. Accordingly, the exchange exploits complementary properties of different offers to find a suitable allocation, for instance, via combinatorial auctions (Caplice \& Sheffi, 2006). Within the domain of trading on freight exchanges, several studies have investigated similar problems that incorporate routing: Song \& Regan (2003) propose an approximation algorithm that searches feasible routes for forming optimal bids. A similar problem with up to 100 requests is solved by Schönberger \& Kopfer (2005) using a memetic algorithm.

All of the previous papers address a problem that is similar to our PPDSP, although none is identical. Therefore, we propose the novel PPDSP as an intriguing topic for operations research. Accordingly, this problem also requires new means for solving it. 


\section{The Profit-Maximizing Pickup and Delivery Selection Problem (PPDSP)}

In this section, we introduce the notation and assumptions of the ProfitMaximimizing Pickup and Delivery Selection Problem (PPDSP) in a mixed-integer setup.

\subsection{Assumptions}

The Profit-Maximizing Pickup and Delivery Selection Problem is based on the following assumption:

- Profit-maximizing carriers with selective pickup. The carrier only transports profitable requests. Hence, there is no obligation to deliver all available requests.

- Less-than-truckload shipping. The carrier can consolidate different requests in the same trip as long as the requests fit into the delivery schedule.

- Independent requests. A certain revenue is assigned to each request, determined by its attributes, such as the distance between the origin and destination, as well as the load. There is no precedence or routing requirements between different requests.

- Heterogeneous fleet with capacity constraint. The carrier owns a fleet of heterogeneous vehicles. Each vehicle has a different capacity and different traveling costs for the same route.

- Time windows and bounded total trip time. Pickup and deliveries must be made within specified time windows. In addition, the travel time between two locations is proportional to the route distance. The total trip time is bounded to reflect legal constraints on driving hours.

\section{2. $P P D S P$ in mixed-integer formulation}

The following PPDSP is based on a mixed-integer formulation of the PDP with time windows (Ropke \& Cordeau, 2009). However, we change the objective function to maximize profits and relax the full delivery constraint for selective pickup.

A total of $n$ transportation requests are represented as a directed graph $G=(V, E)$, whose node set $V=\{0, \ldots, 2 n+1\}$ is divided into nodes $P=\{1,2, \ldots, n\}$ for pickup and nodes $D=\{n+1, n+2, \ldots, 2 n\}$ for delivery, as well as two depots 0 and $2 n+1$ which indicate the starting and final position of a route. Hence, we formalize a request $i$ by using the combination $(i, i+n)$ to refer to the pickup and delivery location. By avoiding the exact geographical coordinates, we simplify the representation, which allows for a better encoding of precedence. For instance, consider two requests $r_{A}=$ $(1,3)$ and $r_{B}=(2,4)$. Then, a route 1-2-4-3 means that the carrier picks up request

$r_{A}$ before request $r_{B}$, but delivers request $r_{B}$ first and request $r_{A}$ last. Finally, delivering request $i$ yields a revenue $\pi_{i}$. 
The carrier operates heterogeneous vehicles $K$, where each vehicle $k \in K$ entails a maximum capacity $Q^{k}$. We then introduce a binary decision variable $x_{i j}^{k}$ that equals 1 if vehicle $k$ travels from node $i$ to node $j$. Such a journey incurs travel $\operatorname{costs} c_{i j}^{k}$ and takes time $t_{i j}^{k}$. Furthermore, each node $i \in V$ must be visited within a time window $\left[l_{i}, u_{i}\right]$. A visit then requires a certain time $d_{i}$ to process, while a capacity $q_{i}$ is loaded or unloaded. Subsequently, a vehicle $k$ leaves a node $i$ at time $v_{i}^{k}$ with new load $w_{i}^{k}$. Finally, the total trip time must be less than $T$. Altogether, this gives the PPDSP formulation as follows:

$$
\max _{x_{i j}^{k}} \sum_{k \in K}\left(\sum_{i \in P} \pi_{i} \sum_{j \in V} x_{i j}^{k}\right)-\sum_{k \in K} \sum_{i \in V} \sum_{j \in V} c_{i j}^{k} x_{i j}^{k}
$$

subject to

$$
\begin{array}{ll}
x_{i j}^{k} \in\{0,1\} & \forall k \in K, i, j \in V \\
\sum_{i \in V} x_{0, j}^{k}=\sum_{i \in V} x_{i, 2 n+1}^{k}=1 & \forall k \in K \\
\sum_{k \in K} \sum_{j \in V} x_{i, 2 n+1}^{k} \leq 1 & \forall k \in K, i \in P \\
\sum_{j \in V} x_{i j}^{k}-\sum_{j \in V} x_{i+n, j}^{k}=0 & \forall k \in K, i \in P \\
\sum_{j \in V} x_{i j}^{k}-\sum_{j \in V} x_{j i}^{k}=0 & \forall k \in K, i \in P \cup D \\
v_{j}^{k} \geq\left(v_{i}^{k}+t_{i j}^{k}+d_{i}\right) x_{i j}^{k} & \forall k \in K, i, j \in V \\
v_{i}^{k}+t_{i, i+n}^{k} \leq v_{i+n}^{k} & \forall k \in K, i, j \in V \\
l_{i} \leq t_{i}^{k} \leq u_{i} & \forall k \in K, i \in V \\
v_{2 n+1}^{k}-v_{0}^{k} \leq T & \forall k \in K \\
w_{j}^{k} \geq\left(w_{i}^{k}+q_{j}\right) x_{i j}^{k} & \forall k \in K, i, j \in V \\
\max \left\{0, q_{i}\right\} \leq w_{i}^{k} \leq \min \left\{Q^{k}, Q^{k}+q_{i}\right\} & \forall k \in K, i \in V
\end{array}
$$

The objective function in Equation (1) maximizes the total profit of all trips, which equals the total revenue from all deliveries minus the total travel cost. The first half yields revenue $\pi_{i}$ if the vehicle visits any pickup node $i \in P$, while the second half sums over all route costs $c_{i j}^{k}$ if edge $(i, j)$ is traversed by vehicle $k$. The bounding of the PPDSP might not be obvious at first glance as extending the route can increase costs at first, but might enable additional deliveries that result into higher revenues and profits later.

Equation (2) introduces the binary decision variable. What follows are constraints regarding the connectivity and precedence of nodes in Equations (3) to (6): Equation (3) ensures that all routes start and end at the depots, whereas Equation (4) 
requires each node $i$ to be visited not more than once. Equation (5) ensures pickup and delivery through the same vehicle, while implicitly defining node $i$ as pickup and $i+n$ as delivery. Next, Equation (6) makes sure that all vehicles only leave non-depot nodes.

Equations (7) to (9) consider time windows as follows: Equation (7) states that the departure time at node $j$ must be later than the departure time at node $i$ plus travel and processing time if route $(i, j)$ is traversed. Note that this is formalized as an inequality constraint to allow for vehicles waiting. In addition, Equation (8) supposes the triangle inequality; it specifies the arrival time at a destination to be at least the time of departure from the corresponding pickup node plus the travel time. However, pickup and delivery need to occur during certain time windows as given in Equation (9). Altogether, Equations (7) to (9) eliminate sub-tours. Furthermore, Equation (10) limits the total time of all journeys.

Additional constraints address the load. Among these is Equation (11), which specifies the load change from node $i$ to node $j$. As the capacity change equals the request's load for pickup nodes and is negative for delivery nodes, Equation (12) requires the vehicle load to be within $\left[q_{i}, Q^{k}\right]$ for pickup nodes and $\left[0, Q^{k}+q_{i}\right]$ for delivery nodes.

The general formulation of the PPDSP is NP-hard. For simplicity, one can assume a single vehicle and arbitrarily large time windows. In addition, consider that the revenues from each request are sufficiently high in comparison to travel costs that are sufficiently low. Then, the problem is essentially a multi-constrained backpack optimization and, as such, NP-hard.

\section{Optimization methods}

This section develops different methods to solve the PPDSP; their relationship is shown in Figure 2. Accordingly, we first propose a simple graph search (Section 4.1) that directly enumerates feasible routes fulfilling the pickup and delivery constraints. These are processed by branch-and-bound to find an optimal solution. For faster performance, we develop a randomized search heuristic for the single-vehicle case. This is then extended into a greedy heuristic for the multi-vehicle case (Section 4.2). Finally, we consider fleets by introducing a Maximum Set Packing formulation in Section 4.3.

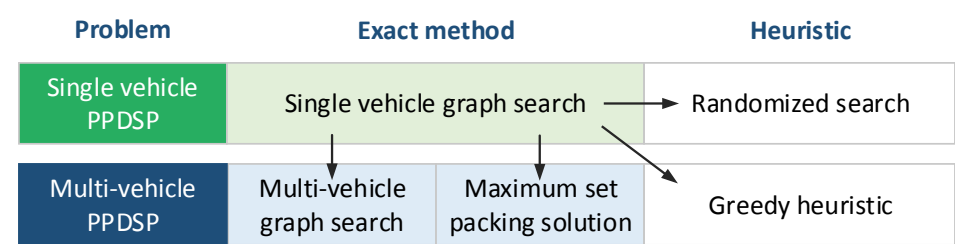

Figure 2: Relationship between problem types and optimization methods. 
Even though the PPDSP resembles other problems from the logistics domain at first glance, several characteristics make it challenging - or even impossible - to apply methods from the literature. For instance, Ropke \& Cordeau (2009) address the PDP with time windows, which requires that all requests are served. In contrast, the selection problem in PPDSP identifies nodes, where a driver might face an unprofitable node $i$, but which brings the driver in the proximity to a very profitable other node. Therefore, one would need to enumerate all feasible subsets and then apply the methodology from Ropke \& Cordeau (2009) - which makes the naïve version of column generation computationally expensive. Similarly, the objective can entail local minima and, hence, the notion of dominance as a requirement for a labeling approach does not hold.

\subsection{Graph search and randomized search for the single vehicle PPDSP}

As a route is represented by an integer sequence, the graph search aims to enumerate all feasible permutations that satisfy the constraints given by Equations (3) to (6). Hence, we use unsorted sets to denote the unvisited pickups $\tilde{P}$ and the onboard requests $\tilde{D}$, while an ordered list $\tilde{R}$ stores the current route. At each stop, the following three options are possible moves for the carrier. We model these via list operations as follows:

1. Pick up a new request $i$. Remove pickup node $i$ from $\tilde{P}$ and add node $i+n$ to $\tilde{D}$ for future delivery; also append node $i$ to $\tilde{R}$ for updating the route.

2. Deliver an onboard request $j$. Remove $j$ from $\tilde{D}$ and append node $j$ to $\tilde{R}$ as part of updating the route.

3. Vehicle empty and return. Check if set of deliveries $\tilde{D}$ is empty and, in that case, append the depot $2 n+1$ to $\tilde{R}$.

In order to translate the above operations into an algorithm, we model these as three branches in a depth-first search. This generates routes that contain no sub-tours, while it maintains the one-to-one correspondence of pickups and deliveries (i. e. each delivery follows its corresponding pickup).

The above depth-first search is bounded by several conditions. For instance, both time and capacity enforce constraints that stop the traversal of a path in the graph search. As part of the implementation, these constraints can be easily verified by looking at a prospective node and the previous route $\tilde{R}$. At the same time, we can allow for waiting times by comparing the arrival time with the permitted time window. Hence, if a vehicle arrives at a node too early, we automatically add a waiting period to the previous stop if feasible. Based on the unvisited nodes $\tilde{P}$, we can also calculate the maximal potential revenue for the remaining nodes and break the search tree early if this does not exceed the current optimum. 
Accordingly, our implementation prunes inferior routes, i.e. where the potential revenue remains below the current best revenue. ${ }^{3}$ In practice, we perform pruning on-the-fly as follows: as the recursion traverses the search tree in a depth-first order, this quickly provides an estimate of potential solution values. Here, we store their maximum in a global variable. We can then check several criteria at the beginning of each recursive call and, having done this, can decide to stop the recursion early. In detail, we test the following conditions:

1. Time windows. We verify that the time windows from Equation (9) are not violated. In addition, we also control for the arrival and departure times as required by Equations (7) and (8).

2. Maximum capacity. If the new requests makes the total load exceed the capacity limit, the recursion is stopped at this point.

3. Comparison to current-best solution. We calculate the travel costs for the current route plus the profits from each requests. In addition, we also compute the theoretical profit from handling all remaining requests that are still available. If this theoretical value remains below the current-best solution from the global variable, we abort the recursion here.

The runtime can depend on the ordering of the visited nodes. We can effortlessly compare different variants by simply changing $\tilde{D}$ to a sorted queue or a stack instead of a list. This e. g. corresponds to the special cases of first-in-first-out (FIFO) or lastin-first-out (LIFO) ordering (Carrabs et al., 2007).

Due to the NP-hardness of PPDSP, finding the exact solution can be costly. Hence, we develop a heuristic in the form of a randomized search that utilizes the previous graph search as a local search. This performs the following operations within a predefined runtime: first, it randomly generates multiple subsets of unvisited pickup nodes $\tilde{P}$ by picking random numbers between 1 and $n$. Then, it uses the graph search to find the best routes within these subsets. In addition, we keep a tabu list for all searched subsets in order to prevent repeated searches. For a problem size larger than 100 requests, we limit the subset size to a range of 10 to 50 requests in order to guarantee that we find at least one feasible solution within a reasonable time.

We have tested different ways of choosing the subsets for the randomized search by, for example, picking high revenue requests with a higher probability or segmenting the whole set into disjoint subsets and applying graph search to each of them. None of these has resulted in measurable improvements over the nave design. ${ }^{4}$

\footnotetext{
${ }^{3} \mathrm{~A}$ common technique in similar problems is column generation (Desrosiers \& Lübbecke, 2005), such as used in the Resource Constrained Shortest Paths Problem (Engineer et al., 2011) or the Pickup and Delivery Problem with Time Windows (Ropke \& Cordeau, 2009). We eschew such a technique as it does not directly integrate in the selection mechanism of our problem; however, this might be a intriguing agenda for future research.

${ }^{4}$ One possible reason may be that these additional operations make the method more complicated and, as such, reduce the number of visited nodes in a fixed runtime limit.
} 


\subsection{Graph search and greedy heuristic for the multi-vehicle PPDSP}

The previous graph search can be extended to the multi-vehicle case by adding a vehicle counter and an additional branch after a vehicle finishes a trip and returns to the depot. The pseudo code with $k$ vehicles is given below in a recursive fashion; we later also detail how it is called and how we retrieve the solution.

This algorithm utilizes the two global variables $R_{\max }$ and $\Pi_{\max }$ to keep track of the current-best route and its objective function. At the same time, these two variables return the final result of the graph search. In addition, the recursive function takes four parameters as input, namely, a list $\tilde{P}$ of available requests, a list of requests $\tilde{D}$ that were picked up and need to be delivered, the current route and $\tilde{R}$ and an integer $k$. The integer $k$ decreases one-by-one with every vehicle that has been processed. Then, the graph search proceeds as follows: it first traverses all possible combinations of routes and requests. At each point with an empty list $\tilde{D}$ of outstanding deliveries, the algorithm assumes the work of the current vehicle to be finished and then generates a route for the next vehicle $k-1$. Accordingly, we see a corresponding call to GRAPHSEARCH in Step 25 for the next remaining vehicle.

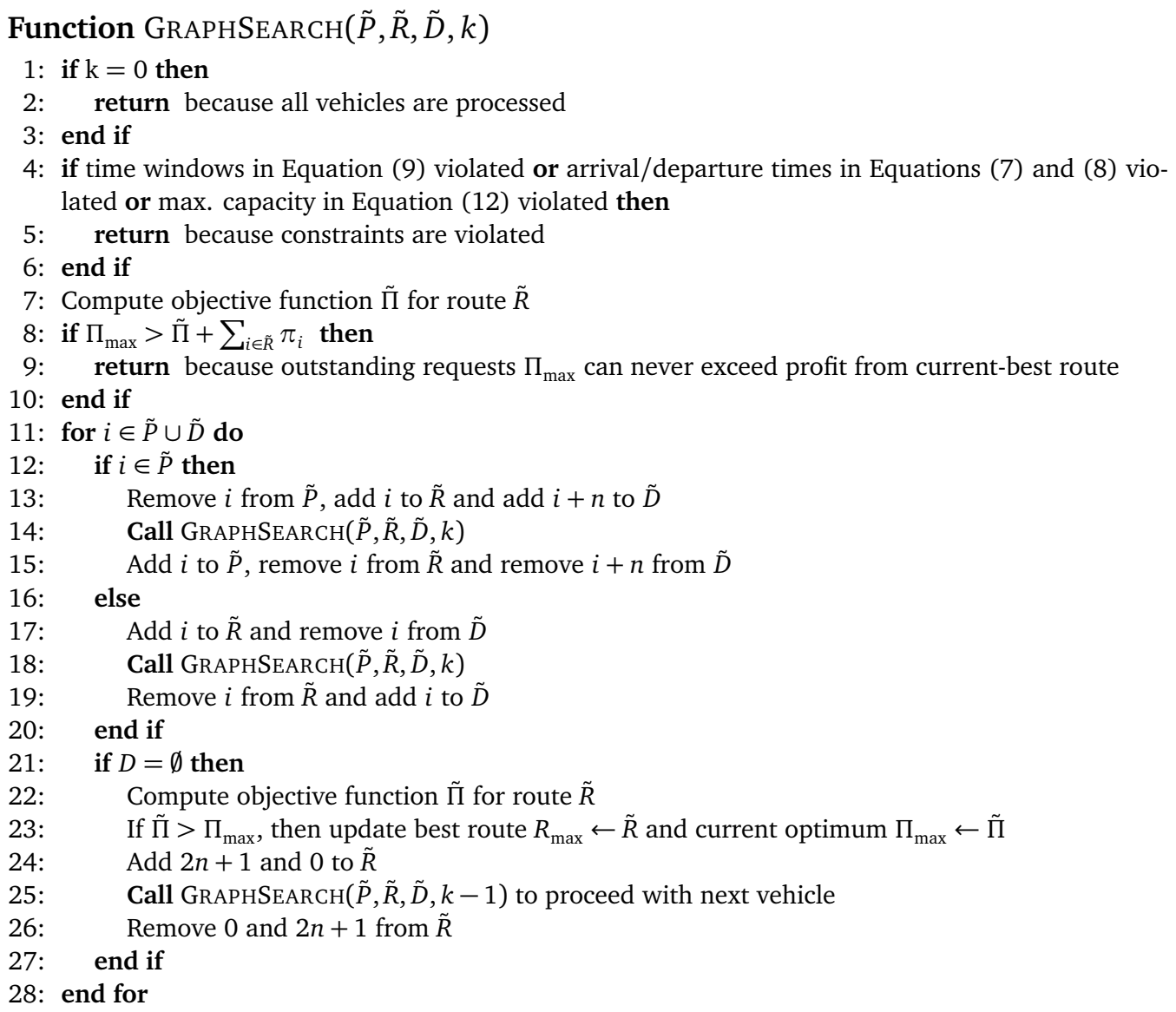


After having defined GRAPHSEARCH, we now specify initial function call. For this purpose, we require two specific values, namely, the number of requests $n$ and the number of vehicles $|K|$. Based on this, we generate initial lists for open requests, pickups and deliveries in order to call GRAPHSEARCH in Step 6 of the following pseudo code snippet. We also initialize the global variables in Steps 4 and 5.

\section{Setup}

1: Initialize $\tilde{P} \leftarrow[1, \ldots, n]$ to keep track of available requests

2: Initialize $\tilde{D} \leftarrow \emptyset$, i. e. requests that were picked up and need to be delivered

3: Initialize list $\tilde{R} \leftarrow[0]$ to store route

4: Store global variable $R_{\max } \leftarrow \emptyset$ to store the current-best route

5: Store global variable $\Pi_{\max } \leftarrow 0$ to store objective function from current-best route

6: Call function GraphSEARCh $(\tilde{P}, \tilde{R}, \tilde{D},|K|)$

When the recursion has traversed its call tree, it stops and yields two global variables storing the optimum as follows.

\section{Results}

1: Route $R_{\max }$ stores order of requests for the optimum

2: Corresponding objective function is given by $\Pi_{\max }$

The algorithm might generate routes such as, for example, $\underbrace{0245}_{k=1}-\underbrace{0135}_{k=2}$ and $\underbrace{0135}_{k=1}$ $\underbrace{0245}_{k=2}$ as two different routes. In the first route, vehicle $k=1$ starts at depot 0 and then visits nodes 2 and 4 before reaching the final depot 5 , while vehicle $k=2$ shares the same initial and instead depots but further visits nodes 1 and 3 . Both routes seem to be interchangeable at first glance. However, this is not the case with heterogeneous fleets where the properties of vehicle $k=1$ and $k=2$ differ: parameter $k$ specifies the vehicle and is counted in descending order.

Our tests indicate that the graph search can quickly solve the single vehicle case but requires a much longer runtime for multi-vehicle cases. Given NP-hardness, we develop the following greedy heuristic for large instances with multiple vehicles as follows:

- First, initialize $\tilde{P}$ with all requests and perform a single vehicle graph search to find the first-best solution.

- Remove the delivered requests from $\tilde{P}$ and search for the best route with the remaining requests.

- Repeat the previous step until all vehicles are used or no more profitable requests are available. 


\subsection{Maximum set packing formulation for homogeneous fleets}

We now propose a method that is tailored to homogenous fleets. In these cases, we obtain routes that are equivalent, such as 0245-0135 and 0135-0245. To avoid duplications, we solve a Maximum Set Packing problem (MSP). Let's assume that the feasible route $r_{m} \in R$ generates a profit $\pi_{m}$ for all feasible routes $m$. Then, we introduce an indicator variable $b_{m i}$ that equals to one if request $i \in P$ is covered. Furthermore, the binary variable $y_{m}$ equals to one if the route is chosen. The MSP is given by

$$
\max _{y_{m}} \sum_{m} \pi_{m} y_{m}
$$

subject to

$$
\begin{array}{ll}
y_{m} \in\{0,1\} & \forall m, \\
\sum_{m} y_{m} \leq|K| & \forall m, \\
\sum_{m} y_{m} b_{m i} \leq 1 & \forall m \forall i \in P,
\end{array}
$$

where $K$ is the number of vehicles in the fleet. The MSP is a well-studied problem that is also NP-hard. We solve it using the Gurobi optimizer (Gurobi, 2014) because of its high performance. We note that it can also be solved using other solvers or a branch-and-bound algorithm.

The MSP solution can be further extended to the case of multiple vehicles of several types such as, for example, the two-type ambulances case - studied in Parragh et al. (2012). Instead of solving the single vehicle PPDSP once for feasible routes, we need to solve a set of profitable routes for each type of vehicle, and then solve the MSP for the profitable routes. In both cases of single and multiple vehicles, one generates the set of feasible routes using graph search. For this purpose, we need to adapt the graph search from Section 4.1 as follows. Our original variant performs pruning in order to remove routes that are inferior to the current best. For the MSP solution, we remove pruning of sub-optimal solutions in order to generate all routines as part of the feasible set.

\section{Computational experiments}

This section executes various computational experiments in order to investigate the quality and performance of the previous optimization strategies. Accordingly, we compare the heuristics with the exact methods (including the Gurobi optimizer) as our benchmark.

Our computational experiments show that the graph search is able to obtain the same optimal solution as the Gurobi optimizer but, in the single vehicle case, over 100 times faster. Our proposed heuristics find highly efficient solutions within seconds; 
all match the performance of methods for similar problem formulations. Finally, we investigate both homogeneous and heterogeneous fleets, where we utilize the Maximum Set Packing model to accelerate the performance.

\subsection{Experimental setup}

We test the previous methods with random instances of requests, where all parameters are sampled from uniform distributions as follows. We generate 10 random instances for each number of requests and compare different combinations of revenue and time windows: (a) regular and (b) high revenue, (c) large time windows and (d) high revenue with large time windows. For consistency, we use the same types of instances across all four settings but vary the values of revenue and time windows. We do not compare different load and capacity settings, as they have an impact that is very similar to time windows.

The nodes are randomly placed on a $[0,1] \times[0,1]$ Euclidean area. Distances are then calculated and scaled up to form the route costs. Request revenues are generated based on the direct route costs and load, plus variations in the form $\pi_{i}=\alpha c_{i, i+n} q_{i}+\delta_{i}$, with $\delta_{i}$ following uniform distribution between $[20,100]$ for low revenue instances and $[60,300]$ for high revenue instances. Furthermore, travel time between nodes is

generated based on route costs plus variations $t_{i j}^{k}=0.1 c_{i j}^{k}+\varepsilon_{i j}^{k}$, with $\varepsilon_{i j}^{k}$ following uniform distribution between $[-1,1]$. Based on the travel time, time windows are generated such that the direct delivery of the request is always feasible. The size of the time windows, loading times and request loads are all generated following uniform distribution. The settings with large time windows simply double the size of the time windows. Finally, each vehicle has a capacity of $7.5 \mathrm{t}$ and the total ride time is set to $56 \mathrm{~h}$. The parameters are summarized in Table 2.

\begin{tabular}{|c|c|c|c|c|c|c|c|c|c|}
\hline \multirow{2}{*}{ Parameters } & \multicolumn{2}{|c|}{ Revenue } & \multirow{2}{*}{$\begin{array}{l}\text { Route } \\
\text { cost }\end{array}$} & \multirow{2}{*}{$\begin{array}{c}\text { Travel } \\
\text { time }\end{array}$} & \multicolumn{2}{|c|}{ Time window } & \multirow{2}{*}{$\begin{array}{l}\text { Loading } \\
\text { time }\end{array}$} & \multirow{2}{*}{$\begin{array}{c}\text { Request } \\
\text { load }\end{array}$} & \multirow{2}{*}{$\begin{array}{c}\text { Total } \\
\text { ride time }\end{array}$} \\
\hline & Low $(\alpha=0.2)$ & High $(\alpha=0.6)$ & & & Regular & Large & & & \\
\hline Lower bound & $€ 30$ & $€ 90$ & $€ 50$ & $0.5 \mathrm{~h}$ & $2 \mathrm{~h}$ & $4 \mathrm{~h}$ & $\mathrm{Oh}$ & $1 \mathrm{t}$ & $56 \mathrm{~h}$ \\
\hline Upper bound & $€ 700$ & $€ 2100$ & $€ 400$ & $20 \mathrm{~h}$ & $10 \mathrm{~h}$ & $20 \mathrm{~h}$ & $20 \mathrm{~h}$ & $7.5 \mathrm{t}$ & $56 \mathrm{~h}$ \\
\hline
\end{tabular}

Table 2: Parameters for the random instances as part of computational experiments.

We implement our proposed optimization methods in C\# with multi-threading. The runtime limit for both graph search and Gurobi is 90 minutes. The experiments are performed on an Intel Core i5-2400 at $3.10 \mathrm{GHz}$ and $2 \mathrm{~GB}$ RAM. For each setup, we average results from 10 random instances.

\subsection{Configuration of Gurobi optimizer}

For comparison, the model is also implemented in the Gurobi optimizer (Gurobi, 2014) via a C\# interface. For performance reasons, we incorporate additional constraints that do not change the solution but accelerate the search. Accordingly, we 
add

$$
\begin{array}{ll}
x_{i j}^{k}=0 & \forall i \in V, \\
x_{0, i}^{k}=0 & \forall i \in D, \\
x_{i, 2 n+1}^{k}=0 & \forall i \in P, \\
x_{i, 0}^{k}=0 & \forall i \in P \cup D, \\
x_{2 n+1, i}^{k}=0 & \forall i \in P \cup D .
\end{array}
$$

Equation (17) eliminates cycles at the nodes, while Equation (18) forbids the direct visit of delivery nodes from the depot. Furthermore, Equation (19) avoids an immediate return to the depot after visiting a pickup node, as well as ever visiting the start depot again in Equation (20). Finally, Equation (21) prevents vehicles from starting their route at the end depot. In addition, the objective, i.e. the profit, is set to non-negative. ${ }^{5}$

\subsection{Solution quality in the single vehicle case}

This section presents comparisons in the single vehicle case across three methods: the graph search (GS) as an exact method, the randomized search (RS) heuristic and the Gurobi optimizer as a benchmark. We evaluate the randomized search with two different limits for the runtime, i. e. $0.5 \mathrm{~s}$ and $2 \mathrm{~s}$. A runtime analysis is provided later in Section 5.4 .

Table 3 compares the profits of the best routes found by the different methods. In the case of the graph search, we report the optimal profits, while we compute relative ratios out of the optimal profits for all other methods in order to study how well the different heuristics approximate the optimal solution. Not all results are reported for the Gurobi optimizer, since several instances exceeded the runtime limit of $90 \mathrm{~min}$. This also explains the far-from-optimal results in cases with large time windows. Altogether, the results reveal that the randomized search performs well for small instances, while the graph search is highly efficient overall.

\footnotetext{
${ }^{5}$ We set the Gurobi parameters as follows: we enable lazy constraints, silence output from the solver process and limit the memory to a threshold of $700 \mathrm{MB}$, beyond which the intermediate results are written to files. Automatic tuning of Gurobi does not yield parameters with consistent performance improvements.
} 


\begin{tabular}{|c|c|c|c|c|c|c|c|c|}
\hline \multicolumn{9}{|c|}{ Profit comparison for regular time windows (in \% of theoretical maximum) } \\
\hline & \multicolumn{4}{|c|}{ (a) Regular revenues } & \multicolumn{4}{|c|}{ (b) High revenues } \\
\hline \#Requests & GS & Gurobi & RS (0.5 s) & RS (2s) & GS & Gurobi & RS (0.5 s) & RS (2s) \\
\hline 40 & 211.6 & $100 \%$ & $100 \%$ & $100 \%$ & 1170.8 & $100 \%$ & $100 \%$ & $100 \%$ \\
\hline 50 & 142.0 & $100 \%$ & $100 \%$ & $100 \%$ & 892.2 & $100 \%$ & $100 \%$ & $100 \%$ \\
\hline 60 & 225.4 & $100 \%$ & $100 \%$ & $100 \%$ & 1293.6 & $100 \%$ & $100 \%$ & $100 \%$ \\
\hline 80 & 176.8 & $100 \%$ & $100 \%$ & $100 \%$ & 1064.4 & $100 \%$ & $100 \%$ & $100 \%$ \\
\hline 100 & 185.2 & $100 \%$ & $100 \%$ & $100 \%$ & 1247.0 & $97 \%$ & $100 \%$ & $100 \%$ \\
\hline 200 & 353.0 & & $100 \%$ & $100 \%$ & 1695.2 & & $100 \%$ & $100 \%$ \\
\hline 300 & 460.2 & & $89 \%$ & $100 \%$ & 2219.0 & & $90 \%$ & $100 \%$ \\
\hline 400 & 585.6 & & $79 \%$ & $96 \%$ & 2641.2 & & $85 \%$ & $93 \%$ \\
\hline 500 & 471.6 & & $90 \%$ & $94 \%$ & 2247.2 & & $93 \%$ & $93 \%$ \\
\hline \multicolumn{9}{|c|}{ Profit comparison for large time windows (in \% of theoretical maximum) } \\
\hline & \multicolumn{4}{|c|}{ (c) Regular revenues } & \multicolumn{4}{|c|}{ (d) High revenues } \\
\hline \#Requests & GS & Gurobi & RS (0.5 s) & RS (2s) & GS & Gurobi & RS $(0.5 \mathrm{~s})$ & RS (2s) \\
\hline 35 & 165.4 & $85 \%$ & $100 \%$ & $100 \%$ & 1297.8 & $98 \%$ & $100 \%$ & $100 \%$ \\
\hline 40 & 339.0 & $72 \%$ & $100 \%$ & $100 \%$ & 1824.6 & $73 \%$ & $100 \%$ & $100 \%$ \\
\hline 50 & 356.6 & $59 \%$ & $100 \%$ & $100 \%$ & 1735.6 & $63 \%$ & $100 \%$ & $100 \%$ \\
\hline 60 & 447.6 & & $100 \%$ & $100 \%$ & 2144.0 & & $100 \%$ & $100 \%$ \\
\hline 80 & 401.2 & & $98 \%$ & $100 \%$ & 2013.0 & & $100 \%$ & $100 \%$ \\
\hline 100 & 423.4 & & $99 \%$ & $100 \%$ & 2161.2 & & $98 \%$ & $100 \%$ \\
\hline 200 & 774.6 & & $80 \%$ & $82 \%$ & 3268.2 & & $89 \%$ & $80 \%$ \\
\hline 300 & 810.4 & & $64 \%$ & $75 \%$ & 3399.4 & & $78 \%$ & $84 \%$ \\
\hline 400 & 938.4 & & $58 \%$ & $69 \%$ & 3826.8 & & $65 \%$ & $70 \%$ \\
\hline
\end{tabular}

Table 3: Profit comparison across different methods for single vehicle instances as a ratio to the profit of the optimal solution. Cells are colored according to this ratio (the darker the shade, the closer the computed solution is to the optimal value). Cases with no percentage reported (white background) exceed the maximum runtime of $90 \mathrm{~min}$. In addition, the column labeled GS (based on the results from the graph search) contains the actual profit in $€$.

\subsection{Runtime in the single vehicle case}

Table 4 compares the runtimes (in seconds) in the single vehicle case for two methods, namely, graph search and the Gurobi solver. In the cases with missing values, we aborted the computation before an optimal solution was reached as a way of enforcing a runtime limit of $90 \mathrm{~min}$. We see that GS is over 1,000 times faster for the single vehicle cases and finds the exact solution quickly for instances with fewer than 100 requests. Within the same runtime limit, GS is able to optimally solve instances with 10 times the number of requests as the Gurobi optimizer. 


\begin{tabular}{|c|c|r|r|r|r|r|r|c|}
\hline & \multicolumn{4}{|c|}{ Regular time windows } & \multicolumn{4}{c|}{ Large time windows } \\
\hline & (a) Regular revenue & \multicolumn{2}{|c|}{ (b) High revenue } & \multicolumn{2}{|c|}{ (c) Regular revenue } & \multicolumn{2}{c|}{ (d) High revenue } \\
\hline \# Requests & \multicolumn{1}{|c|}{ GS } & \multicolumn{1}{|c|}{ Gurobi } & GS & Gurobi & GS & Gurobi & GS & Gurobi \\
\hline 35 & 0.000 & 0.93 & 0.000 & 2.26 & 0.003 & 2160.02 & 0.003 & 3257.61 \\
40 & 0.000 & 3.05 & 0.000 & 6.89 & 0.019 & $(5400.03)$ & 0.019 & $(5400.26)$ \\
50 & 0.002 & 45.17 & 0.002 & 47.02 & 0.043 & $(5400.43)$ & 0.045 & $(5400.05)$ \\
60 & 0.002 & 34.84 & 0.001 & 29.30 & 0.072 & & 0.072 & \\
80 & 0.010 & 266.17 & 0.010 & 297.55 & 0.643 & & 0.644 & \\
100 & 0.016 & 1870.50 & 0.016 & 2166.67 & 0.658 & & 0.563 & \\
300 & 1.490 & & 1.425 & & 549.012 & & 550.793 & \\
500 & 9.259 & & 9.447 & & & & & \\
\hline
\end{tabular}

Table 4: Runtime comparison across different methods for single vehicle instances (in seconds). Instances with numbers in parentheses were forced to stop by the Gurobi runtime limit of 90 min, i. e. 5400 s. All omitted figures also exceeded the runtime limit.

\subsection{Sensitivity analysis in the single vehicle case}

Finally, we perform a sensitivity analysis to identify those parameters which reveal a statistically significant influence on both runtime and profit. For this purpose, we estimate a linear regression model with different dependent variables and then state both the coefficients and $t$-statistics from an ordinary least squares (OLS) estimator. With regard to runtime, we utilize logarithmic values as these better follow a normal distribution. ${ }^{6}$ We also compare models with and without the influence of the revenue variable, as well as investigate the model fit with help of both $F$-statistic and adjusted $R^{2}$. From the results in Table 5, we see that the number of requests, the length of time windows and load show a statistically significant influence on the runtime at common significance levels. However, this changes when observing the profits. Here, the number of requests and load have an non-significant impact.

Since the graph search solves instances with 50 requests in milliseconds, the resulting runtime contains a large share of overhead costs, such as initialization time. Therefore, we test the graph search with an additional set of instances. ${ }^{7}$ We thus estimate a linear model using ordinary least squares and report both OLS coefficients and $t$-statistics in Table 6. As before, we validate the influence on different independent variables, runtime and profit. In addition, both $F$-statistic and adjusted $R^{2}$ compare the fit of the models. The results confirm the exponential increase in runtime with an increasing number of requests, increasing time windows and decreasing request

\footnotetext{
${ }^{6}$ We investigate graph search and the Gurobi optimizer with parameters as follows: instances from 35 to 50 requests in increments of 5 ; three revenue settings of $100 \%, 150 \%$ and $200 \%$; two time windows with one $10 \%$ larger than the other; and three loads ( $80 \%, 90 \%$ and $100 \%)$ of a randomly generated maximum load. We again generate a total of 10 random instances for each setup, giving $4 \times 3 \times 2 \times 3 \times 10=720$ samples. Since the runtime increases exponentially, we take the logarithmic runtime as the dependent variable.

${ }^{7}$ These instances range from 50 to 200 requests in increments of 5 . Revenue, time windows and load have three setups, i. e. $100 \%, 150 \%$ and $200 \%$, of the value specified in Section 5.1. We again generate a total of 10 random instances for each setup, which gives $31 \times 3 \times 3 \times 3 \times 10=8,370$ observations.
} 


\begin{tabular}{lcccccc}
\hline & $\begin{array}{c}\text { Runtime (log.) } \\
\text { graph search }\end{array}$ & $\begin{array}{c}\text { Runtime (log.) } \\
\text { graph search }\end{array}$ & $\begin{array}{c}\text { Runtime (log.) } \\
\text { Gurobi }\end{array}$ & $\begin{array}{c}\text { Runtime (log.) } \\
\text { Gurobi }\end{array}$ & $\begin{array}{c}\text { Profit } \\
\text { graph search }\end{array}$ & $\begin{array}{c}\text { Profit } \\
\text { Gurobi }\end{array}$ \\
\hline Constant & $-11.93^{* *}$ & $-11.97^{* *}$ & $-3.08^{* *}$ & $-2.95^{* *}$ & $-903.54^{* *}$ & $-930.99^{* *}$ \\
& $(-9.50)$ & $(-9.64)$ & $(-7.99)$ & $(-7.73)$ & $(-7.22)$ & $(-7.18)$ \\
Number of requests & $0.16^{* *}$ & $0.16^{* *}$ & $0.06^{* *}$ & $0.06^{* *}$ & -0.48 & -1.31 \\
& $(18.47)$ & $(18.49)$ & $(21.20)$ & $(21.04)$ & $(-0.54)$ & $(-1.42)$ \\
Time windows & $6.26^{* *}$ & $6.26^{* *}$ & $5.45^{* *}$ & $5.45^{* *}$ & $552.03^{* *}$ & $627.33^{* *}$ \\
& $(6.28)$ & $(6.29)$ & $(17.84)$ & $(17.79)$ & $(5.55)$ & $(6.10)$ \\
Load & $-2.93^{* *}$ & $-2.93^{* *}$ & $-1.37^{* *}$ & $-1.37^{* *}$ & 11.29 & -8.56 \\
& $(-4.80)$ & $(-4.81)$ & $(-7.29)$ & $(-7.38)$ & $(0.19)$ & $(-0.14)$ \\
Revenue & -0.02 & & $0.08^{*}$ & & $458.05^{* *}$ & $465.60^{* *}$ \\
& $(-0.20)$ & & $(2.18)$ & & $(37.63)$ & $(36.96)$ \\
\hline$F$-statistic & $100.96^{* *}$ & $134.79^{* *}$ & $205.32^{* *}$ & $270.78^{* *}$ & $361.71^{* *}$ & $351.23^{* *}$ \\
Adjusted $R^{2}$ & 0.36 & 0.36 & 0.53 & 0.53 & 0.67 & 0.66 \\
\hline Stated: OLS coef. and $t$-stat. in parenthesis & & & Stat. significance levels: ${ }^{*} p<5 \%,{ }^{* *} p<1 \%$
\end{tabular}

Table 5: Sensitivity analysis in form of an OLS regression to study which parameters influence both runtime and profit for the exact optimization methods.

loads. These combined explain over $86 \%$ of the variance in the log-runtime. The revenue of the requests only affects the optimal profit but has no significant impact on runtime. 


\begin{tabular}{lccccc}
\hline & $\begin{array}{c}\text { Runtime } \\
\text { graph search }\end{array}$ & $\begin{array}{c}\text { Runtime } \\
\text { graph search }\end{array}$ & $\begin{array}{c}\text { Runtime (log.) } \\
\text { graph search }\end{array}$ & $\begin{array}{c}\text { Runtime (log.) } \\
\text { graph search }\end{array}$ & $\begin{array}{c}\text { Profit } \\
\text { graph search }\end{array}$ \\
\hline Constant & $-2766.81^{* *}$ & $-2803.66^{* *}$ & $-0.85^{* *}$ & $-0.85^{* *}$ & $-1038.51^{* *}$ \\
& $(-5.21)$ & $(-6.20)$ & $(-26.39)$ & $(-30.99)$ & $(-58.85)$ \\
Number of requests & $34.26^{* *}$ & $34.26^{* *}$ & $0.02^{* *}$ & $0.02^{* *}$ & 2.78 \\
& $(20.26)$ & $(20.26)$ & $(150.16)$ & $(150.17)$ & $(49.46)$ \\
Time windows & $3535.09^{* *}$ & $3535.09^{* *}$ & $1.53^{* *}$ & $1.53^{* *}$ & $421.27^{* *}$ \\
Load & $(19.09)$ & $(19.09)$ & $(136.48)$ & $(136.49)$ & $(68.43)$ \\
& $-3584.27^{* *}$ & $-3584.27^{* *}$ & $-1.07^{* *}$ & $-1.07^{* *}$ & $-215.29^{* *}$ \\
Revenue & $(-19.35)$ & $(19.35)$ & $(-94.89)$ & $(-94.90)$ & $(-34.97)$ \\
& -24.57 & & 0.00 & & $737.30^{* *}$ \\
\hline$F$-statistic & $(-0.13)$ & & $(-0.04)$ & & $(119.77)$ \\
Adjusted $R^{2}$ & $287.35^{* *}$ & $383.17^{* *}$ & $12544.81^{* *}$ & $16728.41^{* *}$ & $5674.51^{* *}$ \\
\hline Stated: OLS coef. and $t$-stat. in parenthesis & 0.12 & 0.86 & 0.86 & 0.73 \\
\hline
\end{tabular}

Table 6: Sensitivity analysis in form of an OLS regression to study which parameters influence both runtime and profit for large instances as input to the graph search. The Gurobi optimizer is neglected at this point due to its slow performance.

\subsection{Results of the multi-vehicle case}

We first report results regarding the solution quality for a setup of 2 and 4 vehicles with instances ranging from 10 to 50 requests. We later analyze the Maximum Set Packing solution and the greedy heuristic with up to 500 requests. All results are averaged across 10 random instances.

Table 7 reports the value of the optimal solution, the latter being computed with the help of the graph search. We then compare the optimal value to the solutions found by the other methods as a proportion of the optimum. Both MSP and the greedy heuristic obtain solutions almost identical to the graph search. However, we observe lower values for the Gurobi solver as we enforce a termination after $90 \mathrm{~min}$. In addition, Table 8 compares the runtime, again with an enforced stop after $90 \mathrm{~min}$. Based on both tables, we see that larger time windows allow for more feasible routes, resulting in higher profit and a much longer solution time. More vehicles also significantly increase the difficulty of the solution. Note that for a small number of requests, the optimal solution utilizes only 2 vehicles or less. Therefore, increasing the number of vehicles only improves optimal profits marginally. Interestingly, the greedy heuristic is able to find routes with at least $90 \%$ of the optimal profits.

As for runtime, GS is over 100 times faster than Gurobi, MSP is over twice as fast as GS and the greedy heuristic is about 10 times faster than GS. Gurobi exceeds the 90 minute runtime in 126 out of 200 instances, which leads to sub-optimal routes and low profits.

To further explore the potential of the MSP solution and the greedy heuristic, we test both with 10, 20 and 30 vehicles, while also trying up to 500 requests for regular time windows and up to 400 requests for large time windows. Since MSP is an exact method, we benchmark the efficiency of the greedy heuristic compared 


\begin{tabular}{|c|c|c|c|c|c|c|c|c|c|}
\hline & & \multicolumn{4}{|c|}{2 Vehicles } & \multicolumn{4}{|c|}{4 Vehicles } \\
\hline & \#Requests & GS & Gurobi & MSP & Greedy & GS & Gurobi & MSP & Greedy \\
\hline \multirow{5}{*}{$\begin{array}{l}\text { (a) Regular } \\
\text { revenue }\end{array}$} & 10 & 0.0 & $100 \%$ & $100 \%$ & $100 \%$ & 0.0 & $100 \%$ & $100 \%$ & $100 \%$ \\
\hline & 20 & 121.2 & $100 \%$ & $100 \%$ & $100 \%$ & 121.2 & $100 \%$ & $100 \%$ & $100 \%$ \\
\hline & 30 & 119.8 & $100 \%$ & $100 \%$ & $100 \%$ & 132.0 & $100 \%$ & $100 \%$ & $100 \%$ \\
\hline & 40 & 139.2 & $100 \%$ & $100 \%$ & $100 \%$ & 173.0 & $100 \%$ & $100 \%$ & $100 \%$ \\
\hline & 50 & 229.0 & $100 \%$ & $100 \%$ & $99 \%$ & 258.2 & $97 \%$ & $100 \%$ & $99 \%$ \\
\hline \multirow{5}{*}{$\begin{array}{l}\text { (b) High } \\
\text { revenue }\end{array}$} & 10 & 680.8 & $100 \%$ & $100 \%$ & $100 \%$ & 842.4 & $100 \%$ & $100 \%$ & $98 \%$ \\
\hline & 20 & 1341.6 & $100 \%$ & $100 \%$ & $100 \%$ & 2104.6 & $100 \%$ & $100 \%$ & $100 \%$ \\
\hline & 30 & 1531.6 & $100 \%$ & $100 \%$ & $100 \%$ & 2385.8 & $100 \%$ & $100 \%$ & $100 \%$ \\
\hline & 40 & 1643.6 & $100 \%$ & $100 \%$ & $100 \%$ & 2717.0 & $100 \%$ & $100 \%$ & $100 \%$ \\
\hline & 50 & 1954.8 & $100 \%$ & $100 \%$ & $99 \%$ & 3229.4 & $100 \%$ & $100 \%$ & $99 \%$ \\
\hline \multirow{5}{*}{$\begin{array}{l}\text { (c) Large } \\
\text { time windows }\end{array}$} & 10 & 11.0 & $100 \%$ & $100 \%$ & $100 \%$ & 11.0 & $100 \%$ & $100 \%$ & $100 \%$ \\
\hline & 20 & 291.4 & $100 \%$ & $100 \%$ & $96 \%$ & 277.0 & $93 \%$ & $100 \%$ & $94 \%$ \\
\hline & 30 & 153.4 & $54 \%$ & $100 \%$ & $99 \%$ & 170.2 & $56 \%$ & $100 \%$ & $99 \%$ \\
\hline & 40 & 199.4 & $55 \%$ & $100 \%$ & $100 \%$ & 147.0 & $32 \%$ & $100 \%$ & $93 \%$ \\
\hline & 50 & 70.6 & $15 \%$ & $100 \%$ & $98 \%$ & 57.8 & $9 \%$ & $100 \%$ & $96 \%$ \\
\hline \multirow{5}{*}{$\begin{array}{l}\text { (d) High } \\
\text { revenue } \\
\text { with large } \\
\text { time windows }\end{array}$} & 10 & 925.4 & $100 \%$ & $100 \%$ & $100 \%$ & 1021.8 & $100 \%$ & $100 \%$ & $100 \%$ \\
\hline & 20 & 2236.4 & $100 \%$ & $100 \%$ & $100 \%$ & 3787.6 & $98 \%$ & $100 \%$ & $100 \%$ \\
\hline & 30 & 2461.6 & $80 \%$ & $100 \%$ & $95 \%$ & 3810.8 & $47 \%$ & $100 \%$ & $100 \%$ \\
\hline & 40 & 2675.4 & $59 \%$ & $100 \%$ & $99 \%$ & 4480.4 & $26 \%$ & $100 \%$ & $100 \%$ \\
\hline & 50 & 3028.6 & $27 \%$ & $100 \%$ & $99 \%$ & 5046.8 & $12 \%$ & $100 \%$ & $100 \%$ \\
\hline
\end{tabular}

Table 7: Profit comparison across different methods for multi-vehicle instances as a ratio to the profit of the optimal solution. Cells are colored according to this ratio (the darker the shade, the closer the computed solution is to the optimal value). In addition, the column labeled GS contains the actual profit in $€$.

to the results of MSP in Table 9. More precisely, we denote the greedy value as a ratio out of the exact MSP solution; i. e. a higher ratio shows that the greedy solution better approximates the true optimum. According to Table 9, the greedy heuristic finds solutions with over $86 \%$ of the optimal profits even for very hard instances with 450 requests, 30 vehicles and large time windows. The runtime stays below a minute for instances of up to 450 requests with regular time windows and below an hour for instances of up to 350 requests with large time windows (see Table 10 for runtimes in seconds without maximum execution time).

MSP finds the optimal solution for the homogeneous fleet case using, on average, half the runtime of the greedy heuristic. Breaking down the runtime of MSP reveals that for hard instances, identifying the profitable routes accounts for $99 \%$ of the time, while solving the optimization problem over the routes occupies the other $1 \%$, i.e. in seconds (as shown in Table 11). The overhead costs of passing data to Gurobi and model building are not included in the percentage, which accounts for the remaining 


\begin{tabular}{|c|c|c|c|c|c|c|c|c|c|}
\hline & & \multicolumn{4}{|c|}{2 Vehicles } & \multicolumn{4}{|c|}{4 Vehicles) } \\
\hline & \#Requests & GS & Gurobi & MSP & Greedy & GS & Gurobi & MSP & Greedy \\
\hline \multirow{5}{*}{$\begin{array}{l}\text { (a) Regular } \\
\text { revenue }\end{array}$} & 10 & 0.05 & 0.00 & 0.00 & 0.00 & 0.01 & 0.00 & 0.00 & 0.00 \\
\hline & 20 & 0.09 & 1.26 & 0.03 & 0.00 & 3.44 & 14.10 & 0.00 & 0.00 \\
\hline & 30 & 0.17 & 16.76 & 1.04 & 0.00 & 3.61 & 184.90 & 0.14 & 0.03 \\
\hline & 40 & 0.38 & 32.58 & 0.06 & 0.14 & 3.60 & 508.70 & 0.02 & 0.01 \\
\hline & 50 & 1.22 & 101.72 & 0.28 & 0.06 & 3.61 & 2348.08 & 0.10 & 0.07 \\
\hline \multirow{5}{*}{$\begin{array}{l}\text { (b) High } \\
\text { revenue }\end{array}$} & 10 & 0.00 & 0.09 & 0.01 & 0.00 & 0.01 & 0.20 & 0.00 & 0.00 \\
\hline & 20 & 0.01 & 5.11 & 0.01 & 0.00 & 3.34 & 31.17 & 0.00 & 0.00 \\
\hline & 30 & 0.05 & 22.44 & 0.01 & 0.00 & 3.60 & 793.38 & 0.00 & 0.00 \\
\hline & 40 & 0.11 & 35.70 & 0.08 & 0.00 & 3.60 & 1019.90 & 0.00 & 0.01 \\
\hline & 50 & 0.56 & 119.69 & 0.02 & 0.03 & 3.63 & 2458.60 & 0.39 & 0.11 \\
\hline \multirow{5}{*}{$\begin{array}{l}\text { (c) Large } \\
\text { time windows }\end{array}$} & 10 & 0.43 & 1.13 & 0.37 & 0.05 & 1.00 & 3.56 & 0.30 & 0.02 \\
\hline & 20 & 2.90 & 4635.90 & 0.37 & 0.01 & 3.00 & 2800.00 & 0.27 & 0.01 \\
\hline & 30 & 2.35 & 5400.69 & 0.44 & 0.18 & 3.77 & 5402.01 & 1.31 & 1.24 \\
\hline & 40 & 5.02 & 5400.98 & 0.70 & 0.08 & 4.49 & 5404.55 & 0.70 & 0.44 \\
\hline & 50 & 4.62 & 5401.14 & 0.42 & 0.05 & 3.64 & 5404.16 & 0.88 & 0.14 \\
\hline \multirow{5}{*}{$\begin{array}{l}\text { (d) High } \\
\text { revenue } \\
\text { with large } \\
\text { time windows }\end{array}$} & 10 & 0.00 & 16.67 & 0.00 & 0.00 & 0.86 & 380.14 & 0.05 & 0.01 \\
\hline & 20 & 1.71 & 5045.48 & 0.37 & 0.03 & 3.00 & 3600.00 & 0.51 & 0.02 \\
\hline & 30 & 2.43 & 5400.03 & 0.16 & 0.03 & 3.64 & 5402.65 & 0.76 & 0.67 \\
\hline & 40 & 3.50 & 5400.23 & 0.18 & 0.02 & 3.72 & 5406.42 & 1.41 & 0.29 \\
\hline & 50 & 3.63 & 5400.48 & 0.54 & 0.12 & 3.63 & 5403.04 & 1.08 & 0.16 \\
\hline
\end{tabular}

Table 8: Runtime comparison across different methods for multi-vehicle instances (in seconds). Cells are colored according to this runtime (the darker the shade, the higher the runtime).

percentage of the runtime in easier instances.

Table 12 represents experiments using heterogeneous fleets, where we model a carrier with an equal number of large vehicles ( $7.5 \mathrm{t}$ capacity) and small vehicles $(5.5 \mathrm{t})$. Here, the greedy searches routes for vehicles based on the descending order of their capacity. As before, we state relative numbers as we compare our greedy heuristic with the MSP variant extended to heterogeneous fleets. In summary, both MSP and the greedy heuristic obtain solutions of very similar outcome. In addition, the computational performance of the greedy heuristic resembles the results from the case of a homogeneous fleet as the underlying algorithm remains the same.

Finally, we utilize the PDP100 datasets provided by Li \& Lim (2001) in order to test our methods with real data. ${ }^{8}$ The corresponding results are presented in Table 13, where we compare the profit (and other characteristics) from both the greedy

\footnotetext{
${ }^{8}$ We set the profit for each pickup to $\pi_{j}=€ 10,000$ and the maximum trip time to $T=500$.
} 


\begin{tabular}{|c|c|c|c|c|c|c|}
\hline Regular time windows & \multicolumn{3}{|c|}{ (a) Regular revenue } & \multicolumn{3}{|c|}{ (b) High revenue } \\
\hline \#Requests & 10 Vehicles & 20 Vehicles & 30 Vehicles & 10 Vehicles & 20 Vehicles & 30 Vehicles \\
\hline 50 & $93 \%$ & $93 \%$ & $93 \%$ & $99 \%$ & $99 \%$ & $99 \%$ \\
\hline 100 & $98 \%$ & $98 \%$ & $98 \%$ & $99 \%$ & $98 \%$ & $98 \%$ \\
\hline 150 & $100 \%$ & $99 \%$ & $99 \%$ & $98 \%$ & $99 \%$ & $99 \%$ \\
\hline 200 & $98 \%$ & $97 \%$ & $97 \%$ & $98 \%$ & $97 \%$ & $98 \%$ \\
\hline 250 & $99 \%$ & $97 \%$ & $97 \%$ & $98 \%$ & $98 \%$ & $98 \%$ \\
\hline 300 & $95 \%$ & $94 \%$ & $93 \%$ & $98 \%$ & $97 \%$ & $97 \%$ \\
\hline 350 & $98 \%$ & $97 \%$ & $96 \%$ & $98 \%$ & $98 \%$ & $98 \%$ \\
\hline 400 & $97 \%$ & $95 \%$ & $94 \%$ & $99 \%$ & $97 \%$ & $96 \%$ \\
\hline 450 & $99 \%$ & $97 \%$ & $96 \%$ & $98 \%$ & $98 \%$ & $97 \%$ \\
\hline 500 & $97 \%$ & $96 \%$ & $94 \%$ & $99 \%$ & $98 \%$ & $97 \%$ \\
\hline Large time windows & \multicolumn{3}{|c|}{ (c) Regular revenue } & \multicolumn{3}{|c|}{ (d) High revenue) } \\
\hline \#Requests & 10 Vehicles & 20 Vehicles & 30 Vehicles & 10 Vehicles & 20 Vehicles & 30 Vehicles \\
\hline 50 & $94 \%$ & $94 \%$ & $94 \%$ & $94 \%$ & $94 \%$ & $94 \%$ \\
\hline 100 & $90 \%$ & $88 \%$ & $88 \%$ & $94 \%$ & $93 \%$ & $93 \%$ \\
\hline 150 & $90 \%$ & $87 \%$ & $87 \%$ & $95 \%$ & $94 \%$ & $93 \%$ \\
\hline 200 & $91 \%$ & $86 \%$ & $86 \%$ & $95 \%$ & $94 \%$ & $93 \%$ \\
\hline 250 & $95 \%$ & $90 \%$ & $87 \%$ & $96 \%$ & $95 \%$ & $94 \%$ \\
\hline 300 & $91 \%$ & $90 \%$ & $88 \%$ & $95 \%$ & $94 \%$ & $93 \%$ \\
\hline 350 & $92 \%$ & $90 \%$ & $88 \%$ & $95 \%$ & $94 \%$ & $93 \%$ \\
\hline 400 & $93 \%$ & $91 \%$ & $89 \%$ & $94 \%$ & $93 \%$ & $93 \%$ \\
\hline 450 & $99 \%$ & $97 \%$ & $96 \%$ & $98 \%$ & $98 \%$ & $97 \%$ \\
\hline 500 & $97 \%$ & $96 \%$ & $94 \%$ & $99 \%$ & $98 \%$ & $97 \%$ \\
\hline
\end{tabular}

Table 9: Comparison of the greedy heuristic to the MSP solution for multi-vehicle instances. Performance is given as a ratio to the profit of the solution based on MSP. Cells are colored according to this ratio (the darker the shade, the closer the computed solution is to the optimal value).

heuristic and the MSP approach. We report all instances for which we receive a result within 30 min as many instances are highly complex and represent difficult challenges for routing. Altogether, both MSP and the greedy heuristic again result in solutions of similar magnitude. In addition, we compare the characteristics of the solutions to those of the original but different PDPTW problem. ${ }^{9}$ Evidently, the routines designed for PPDSP only serve a small share of the total requests, while PPDTW requires to serve all of them. By comparing travel time and distance, we cannot observe a clear pattern, as there a multiple instance where PPDSP-designated approaches and PPDTW solutions yield smaller outcomes.

\footnotetext{
${ }^{9}$ Source: https://www.sintef .no/projectweb/top/pdptw/li-lim-benchmark/100customers. Accessed on September 2, 2016.
} 


\begin{tabular}{|c|c|c|c|c|c|c|c|c|c|c|c|c|}
\hline \multirow{3}{*}{\begin{tabular}{|c} 
Regular time windows \\
\#Requests
\end{tabular}} & \multicolumn{6}{|c|}{ (a) Regular revenue } & \multicolumn{6}{|c|}{ (b) High revenue } \\
\hline & \multicolumn{2}{|c|}{10 Vehicles } & \multicolumn{2}{|c|}{20 Vehicles } & \multicolumn{2}{|c|}{30 Vehicles } & \multicolumn{2}{|c|}{10 Vehicles } & \multicolumn{2}{|c|}{20 Vehicles } & \multicolumn{2}{|c|}{30 Vehicles } \\
\hline & Greedy & MSP & Greedy & MSP & Greedy & MSP & Greedy & MSP & Greedy & MSP & Greedy & MSP \\
\hline 50 & 0.0 & 0.2 & 0.0 & 0.0 & 0.1 & 0.0 & 0.0 & 0.0 & 0.0 & 0.0 & 0.0 & 0.0 \\
\hline 100 & 0.1 & 0.1 & 0.2 & 0.0 & 0.3 & 0.0 & 0.1 & 0.0 & 0.1 & 0.0 & 0.1 & 0.0 \\
\hline 150 & 0.3 & 0.1 & 0.5 & 0.1 & 0.6 & 0.1 & 0.3 & 0.1 & 0.4 & 0.1 & 0.4 & 0.1 \\
\hline 200 & 1.3 & 0.3 & 1.9 & 0.3 & 2.4 & 0.3 & 1.2 & 0.3 & 1.5 & 0.3 & 1.7 & 0.4 \\
\hline 250 & 2.8 & 0.6 & 4.0 & 0.6 & 5.0 & 0.6 & 2.5 & 0.6 & 3.3 & 0.6 & 3.7 & 0.6 \\
\hline 300 & 4.1 & 0.9 & 6.1 & 0.8 & 7.7 & 0.8 & 3.6 & 0.9 & 5.1 & 0.9 & 6.0 & 0.8 \\
\hline 350 & 9.6 & 2.0 & 13.7 & 2.0 & 17.0 & 2.0 & 8.0 & 2.1 & 11.1 & 2.0 & 13.1 & 2.0 \\
\hline 400 & 16.2 & 3.2 & 23.3 & 3.0 & 28.0 & 3.2 & 13.1 & 3.0 & 17.1 & 3.2 & 20.0 & 3.3 \\
\hline 450 & 21.0 & 4.2 & 30.9 & 4.1 & 37.5 & 3.9 & 19.9 & 4.0 & 28.6 & 4.0 & 32.6 & 4.0 \\
\hline 500 & 44.5 & 13.6 & 66.3 & 13.8 & 79.6 & 13.6 & 47.2 & 14.4 & 62.6 & 14.0 & 70.8 & 13.8 \\
\hline
\end{tabular}

\begin{tabular}{|c|c|c|c|c|c|c|c|c|c|c|c|c|}
\hline \multirow{3}{*}{$\begin{array}{c}\text { Large time windows } \\
\text { \#Requests }\end{array}$} & \multicolumn{6}{|c|}{ (c) Regular revenue } & \multicolumn{6}{|c|}{ (d) High revenue } \\
\hline & \multicolumn{2}{|c|}{10 Vehicles } & \multicolumn{2}{|c|}{20 Vehicles } & \multicolumn{2}{|c|}{30 Vehicles } & \multicolumn{2}{|c|}{10 Vehicles } & \multicolumn{2}{|c|}{20 Vehicles } & \multicolumn{2}{|c|}{30 Vehicles } \\
\hline & Greedy & MSP & Greedy & MSP & Greedy & MSP & Greedy & MSP & Greedy & MSP & Greedy & MSP \\
\hline 50 & 0.2 & 0.1 & 0.2 & 0.1 & 0.3 & 0.1 & 0.1 & 0.1 & 0.1 & 0.1 & 0.1 & 0.1 \\
\hline 100 & 2.9 & 1.0 & 4.2 & 0.9 & 5.8 & 1.0 & 1.9 & 1.0 & 2.1 & 1.1 & 2.1 & 1.0 \\
\hline 150 & 16.3 & 8.4 & 17.0 & 8.4 & 17.3 & 8.2 & 12.7 & 8.3 & 13.2 & 8.9 & 13.6 & 8.7 \\
\hline 200 & 130.0 & 67.5 & 134.8 & 64.1 & 138.7 & 67.2 & 118.9 & 68.4 & 120.3 & 64.1 & 124.2 & 66.5 \\
\hline 250 & 328.3 & 221.3 & 333.6 & 220.3 & 350.2 & 229.6 & 297.4 & 223.1 & 299.8 & 221.9 & 306.8 & 220.0 \\
\hline 300 & 713.1 & 377.6 & 762.0 & 377.1 & 762.3 & 367.7 & 713.4 & 360.7 & 747.7 & 396.1 & 766.4 & 384.1 \\
\hline 350 & 1868.0 & 1000.6 & 2008.5 & 988.9 & 2000.8 & 968.6 & 1864.9 & 1022.8 & 2135.1 & 1192.3 & 2222.1 & 1094.9 \\
\hline 400 & 3790.7 & 2045.2 & 4076.5 & 2008.4 & 4067.7 & 1964.6 & 3778.2 & 1970.8 & 4148.0 & 2302.9 & 4358.0 & 2137.1 \\
\hline
\end{tabular}

Table 10: Runtime comparison of the greedy heuristic compared to MSP approach for multi-vehicle instances (in seconds). Cells are colored according to this runtime (the darker the shade, the higher the runtime). 


\begin{tabular}{|c|c|c|c|c|c|c|c|c|c|c|c|c|}
\hline \multirow{3}{*}{\begin{tabular}{|c|} 
Regular time windows \\
\# Requests
\end{tabular}} & \multicolumn{6}{|c|}{ (a) Regular revenue } & \multicolumn{6}{|c|}{ (b) High revenue } \\
\hline & \multicolumn{2}{|c|}{10 Vehicles } & \multicolumn{2}{|c|}{20 Vehicles } & \multicolumn{2}{|c|}{30 Vehicles } & \multicolumn{2}{|c|}{10 Vehicles } & \multicolumn{2}{|c|}{20 Vehicles } & \multicolumn{2}{|c|}{30 Vehicles } \\
\hline & GS & MSP & GS & MSP & GS & MSP & GS & MSP & GS & MSP & GS & MSP \\
\hline 50 & $67 \%$ & $0 \%$ & $56 \%$ & $0 \%$ & $68 \%$ & $0 \%$ & $67 \%$ & $0 \%$ & $56 \%$ & $0 \%$ & $68 \%$ & $0 \%$ \\
\hline 100 & $88 \%$ & $0 \%$ & $86 \%$ & $22 \%$ & $88 \%$ & $12 \%$ & $88 \%$ & $0 \%$ & $86 \%$ & $22 \%$ & $88 \%$ & $12 \%$ \\
\hline 150 & $91 \%$ & $4 \%$ & $91 \%$ & $4 \%$ & $91 \%$ & $8 \%$ & $91 \%$ & $1 \%$ & $91 \%$ & $4 \%$ & $91 \%$ & $8 \%$ \\
\hline 200 & $96 \%$ & $3 \%$ & $96 \%$ & $4 \%$ & $97 \%$ & $2 \%$ & $96 \%$ & $3 \%$ & $96 \%$ & $4 \%$ & $97 \%$ & $2 \%$ \\
\hline 250 & $97 \%$ & $2 \%$ & $97 \%$ & $2 \%$ & $97 \%$ & $3 \%$ & $97 \%$ & $2 \%$ & $97 \%$ & $2 \%$ & $97 \%$ & $3 \%$ \\
\hline 300 & $97 \%$ & $2 \%$ & $97 \%$ & $2 \%$ & $96 \%$ & $2 \%$ & $97 \%$ & $2 \%$ & $97 \%$ & $2 \%$ & $96 \%$ & $2 \%$ \\
\hline 350 & $99 \%$ & $0 \%$ & $100 \%$ & $0 \%$ & $100 \%$ & $0 \%$ & $98 \%$ & $2 \%$ & $97 \%$ & $2 \%$ & $97 \%$ & $2 \%$ \\
\hline 400 & $100 \%$ & $0 \%$ & $100 \%$ & $0 \%$ & $100 \%$ & $0 \%$ & $99 \%$ & $1 \%$ & $99 \%$ & $1 \%$ & $99 \%$ & $1 \%$ \\
\hline 450 & $100 \%$ & $0 \%$ & $100 \%$ & $0 \%$ & $100 \%$ & $0 \%$ & $99 \%$ & $1 \%$ & $99 \%$ & $1 \%$ & $99 \%$ & $1 \%$ \\
\hline 500 & $100 \%$ & $0 \%$ & $100 \%$ & $0 \%$ & $100 \%$ & $0 \%$ & $99 \%$ & $1 \%$ & $99 \%$ & $1 \%$ & $99 \%$ & $1 \%$ \\
\hline \multirow{2}{*}{ Large time windows } & \multicolumn{6}{|c|}{ (c) Regular revenue } & \multicolumn{6}{|c|}{ (d) High revenue } \\
\hline & \multicolumn{2}{|c|}{10 Vehicles } & \multicolumn{2}{|c|}{20 Vehicles } & \multicolumn{2}{|c|}{30 Vehicles } & \multicolumn{2}{|c|}{10 Vehicles } & \multicolumn{2}{|c|}{20 Vehicles } & \multicolumn{2}{|c|}{30 Vehicles } \\
\hline \# Requests & GS & MSP & GS & MSP & GS & MSP & GS & MSP & GS & MSP & GS & MSP \\
\hline 50 & $80 \%$ & $19 \%$ & $81 \%$ & $19 \%$ & $82 \%$ & $19 \%$ & $97 \%$ & $3 \%$ & $97 \%$ & $0 \%$ & $97 \%$ & $4 \%$ \\
\hline 100 & $90 \%$ & $10 \%$ & $90 \%$ & $9 \%$ & $88 \%$ & $11 \%$ & $97 \%$ & $2 \%$ & $97 \%$ & $3 \%$ & $98 \%$ & $2 \%$ \\
\hline 150 & $96 \%$ & $3 \%$ & $97 \%$ & $3 \%$ & $96 \%$ & $3 \%$ & $98 \%$ & $2 \%$ & $98 \%$ & $2 \%$ & $98 \%$ & $2 \%$ \\
\hline 200 & $99 \%$ & $1 \%$ & $99 \%$ & $1 \%$ & $99 \%$ & $1 \%$ & $99 \%$ & $0 \%$ & $99 \%$ & $1 \%$ & $99 \%$ & $0 \%$ \\
\hline 250 & $99 \%$ & $1 \%$ & $99 \%$ & $1 \%$ & $99 \%$ & $1 \%$ & $99 \%$ & $1 \%$ & $99 \%$ & $1 \%$ & $99 \%$ & $1 \%$ \\
\hline 300 & $98 \%$ & $1 \%$ & $98 \%$ & $1 \%$ & $98 \%$ & $1 \%$ & $99 \%$ & $1 \%$ & $99 \%$ & $1 \%$ & $99 \%$ & $1 \%$ \\
\hline 350 & $99 \%$ & $1 \%$ & $99 \%$ & $1 \%$ & $99 \%$ & $1 \%$ & $99 \%$ & $1 \%$ & $99 \%$ & $1 \%$ & $99 \%$ & $1 \%$ \\
\hline 400 & $99 \%$ & $1 \%$ & $99 \%$ & $1 \%$ & $99 \%$ & $1 \%$ & $99 \%$ & $1 \%$ & $99 \%$ & $1 \%$ & $99 \%$ & $1 \%$ \\
\hline
\end{tabular}

Table 11: Runtime breakdown of the MSP approach for multi-vehicle instances. The total runtime splits into the ratio spent on the graph search (GS) for finding the profit-maximizing route and solving the Maximum Set Packing (MSP) with Gurobi (plus an additional initialization that is not reported here). Cells are colored according to this runtime (the darker the shade, the higher the runtime). 


\begin{tabular}{|c|r|r|r|r|r|r|}
\hline Regular time windows & \multicolumn{3}{|c|}{ (a) Regular revenue } & \multicolumn{3}{|c|}{ (b) High revenue } \\
\hline \#Requests & 10 Vehicles & 20 Vehicles & 30 Vehicles & 10 Vehicles & 20 Vehicles & 30 Vehicles \\
\hline 50 & $100 \%$ & $100 \%$ & $100 \%$ & $100 \%$ & $99 \%$ & $97 \%$ \\
100 & $100 \%$ & $98 \%$ & $98 \%$ & $99 \%$ & $99 \%$ & $97 \%$ \\
150 & $98 \%$ & $91 \%$ & $89 \%$ & $98 \%$ & $97 \%$ & $96 \%$ \\
200 & $97 \%$ & $94 \%$ & $92 \%$ & $99 \%$ & $98 \%$ & $95 \%$ \\
\hline \hline Large time windows & \multicolumn{2}{|c|}{ (c) Regular revenue } & \multicolumn{2}{|c|}{ (d) High revenue) } \\
\hline \#Requests & 10 Vehicles & 20 Vehicles & 30 Vehicles & 10 Vehicles & 20 Vehicles & 30 Vehicles \\
\hline 50 & $96 \%$ & $83 \%$ & $83 \%$ & $96 \%$ & $93 \%$ & $91 \%$ \\
\hline 100 & $94 \%$ & $83 \%$ & $81 \%$ & $96 \%$ & $93 \%$ & $92 \%$ \\
150 & $91 \%$ & $83 \%$ & $77 \%$ & $95 \%$ & $92 \%$ & $90 \%$ \\
200 & $93 \%$ & $85 \%$ & $78 \%$ & $96 \%$ & $93 \%$ & $89 \%$ \\
\hline
\end{tabular}

Table 12: Comparison of the greedy heuristic to the MSP solution for heterogeneous fleets. While $50 \%$ of the vehicles have a capacity limit of $7.5 \mathrm{t}$, the rest are set to $5.5 \mathrm{t}$. Here, the greedy searches routes for vehicles based on the descending order of their capacity. Performance is given as a ratio to the profit of the optimal solution based on MSP. Cells are colored according to this ratio (the darker the shade, the closer the computed solution is to the optimal value).

\begin{tabular}{|c|c|c|c|c|c|c|c|c|c|c|c|c|c|}
\hline \multirow[t]{2}{*}{ Instance } & \multirow{2}{*}{$\begin{array}{l}\text { Ratio } \\
\text { in } \%\end{array}$} & \multicolumn{5}{|c|}{ Greedy } & \multicolumn{5}{|c|}{ MSP } & \multicolumn{2}{|c|}{ Comparison: PDPTW } \\
\hline & & Profit & Util. & $\begin{array}{c}\text { Served } \\
\text { requests }\end{array}$ & $\begin{array}{c}\text { Non-served } \\
\text { requests }\end{array}$ & $\begin{array}{c}\text { Travel } \\
\text { time }\end{array}$ & Profit & Util. & $\begin{array}{l}\text { Served } \\
\text { requests }\end{array}$ & $\begin{array}{c}\text { Non-served } \\
\text { requests }\end{array}$ & $\begin{array}{c}\text { Travel } \\
\text { time }\end{array}$ & Vehicles & Distance \\
\hline LC101 & 100 & 9929 & 0.005 & 1 & 52 & 251 & 9929 & 0.005 & 1 & 52 & 251 & 10 & 828.94 \\
\hline LC102 & 91 & 99435 & 0.006 & 13 & 40 & 2365 & 109356 & 0.002 & 2 & 51 & 419 & 10 & 828.94 \\
\hline LC103 & 95 & 179160 & 0.014 & 22 & 30 & 4080 & 189162 & 0.006 & 2 & 50 & 392 & 9 & 1035.35 \\
\hline LC105 & 100 & 29867 & 0.004 & 3 & 50 & 673 & 29867 & 0.003 & 2 & 51 & 417 & 10 & 828.94 \\
\hline LC106 & 100 & 49690 & 0.006 & 6 & 47 & 1120 & 49690 & 0.002 & 2 & 51 & 476 & 10 & 828.94 \\
\hline LC107 & 88 & 69607 & 0.009 & 8 & 45 & 1653 & 79496 & 0.009 & 2 & 51 & 436 & 10 & 828.94 \\
\hline LC108 & 100 & 39773 & 0.005 & 4 & 49 & 947 & 39781 & 0.005 & 2 & 51 & 453 & 10 & 828.44 \\
\hline LC109 & 92 & 109497 & 0.010 & 13 & 40 & 2393 & 119462 & 0.006 & 2 & 51 & 405 & 9 & 1000.60 \\
\hline LC201 & 100 & 9966 & 0.001 & 1 & 50 & 214 & 9966 & 0.001 & 1 & 50 & 214 & 3 & 591.56 \\
\hline LC202 & 100 & 59633 & 0.002 & 7 & 44 & 1447 & 59675 & 0.001 & 2 & 49 & 446 & 3 & 591.56 \\
\hline LC203 & 100 & 109229 & 0.002 & 14 & 37 & 2751 & 109306 & 0.001 & 2 & 49 & 423 & 3 & 591.17 \\
\hline LC204 & 89 & 248640 & 0.002 & 31 & 20 & 5770 & 278353 & 0.001 & 2 & 49 & 402 & 3 & 590.60 \\
\hline LC206 & 100 & 9965 & 0.001 & 1 & 50 & 215 & 9965 & 0.001 & 1 & 50 & 215 & 3 & 588.49 \\
\hline LC207 & 100 & 19933 & 0.001 & 2 & 49 & 427 & 19933 & 0.001 & 2 & 49 & 427 & 3 & 588.29 \\
\hline LC208 & 50 & 9936 & 0.001 & 1 & 50 & 244 & 19860 & 0.001 & 1 & 50 & 244 & 3 & 588.32 \\
\hline LR101 & 100 & 49658 & 0.006 & 6 & 47 & 432 & 49658 & 0.004 & 2 & 51 & 147 & 19 & 1650.80 \\
\hline LR105 & 72 & 208846 & 0.014 & 25 & 28 & 1554 & 288270 & 0.005 & 4 & 49 & 200 & 14 & 1377.11 \\
\hline LRC101 & 82 & 178732 & 0.010 & 22 & 31 & 1608 & 218379 & 0.004 & 3 & 50 & 168 & 14 & 1708.80 \\
\hline LRC102 & 89 & 308087 & 0.012 & 37 & 16 & 2503 & 347546 & 0.005 & 4 & 49 & 208 & 12 & 1558.07 \\
\hline LRC105 & 82 & 228524 & 0.010 & 28 & 26 & 1896 & 278083 & 0.005 & 4 & 50 & 197 & 13 & 1637.62 \\
\hline LRC106 & 91 & 298328 & 0.009 & 35 & 18 & 2252 & 328079 & 0.006 & 4 & 49 & 221 & 11 & 1424.73 \\
\hline LRC107 & 83 & 348085 & 0.017 & 41 & 12 & 2595 & 417653 & 0.011 & 4 & 49 & 209 & 10 & 1147.43 \\
\hline
\end{tabular}

Table 13: Comparison of both the greedy heuristic and the MSP solution for homogeneous fleets based on PDP100 data from Li \& Lim (2001). Missing datasets indicate instance where either algorithm exceeds the runtime limit. The ratio compares the profit of both approaches, while further characteristics compare the solution. In addition, we compare it to known solutions of the PDPTW case. 


\subsection{Managerial implications}

Our computer experiments reveal that the difficulty in solving PPDSP comes from the fleet size, the number of available requests, the size of time windows and the request loads. The problem becomes harder to solve optimally when one encounters more requests with larger flexibility in delivery and a smaller ratio of load to vehicle capacity. Then, the runtime increases exponentially with respect to these factors, as these have a significant impact on the optimal profit.

For carriers with a single vehicle, the graph search is sufficient to provide realtime decision support for instances with up to 500 requests under regular settings, or up to 200 requests with large time windows or small request loads in seconds, both of which match the performance of previous studies. For harder instances, the carrier can use graph search on a preferred subset of requests filtered by cargo type, location, distance or revenue or take the Randomized Search heuristic for a speedy solution.

For carriers with vehicle fleets, they can simply use graph search to identify all profitable routes for each vehicle type and then solve a Maximum Set Packing problem for the routes. For carriers with heterogeneous fleets, the graph search can handle instances with up to 4 vehicles and 30 requests within an hour. Harder instances can be solved quickly with the highly efficient greedy heuristic.

\section{Conclusion and Outlook}

In this paper, we studied a novel optimal freight selection problem faced by lessthan-truckload logistics carriers trading on freight exchanges. We modeled it as a profit-maximizing pickup and delivery problem with time windows and selective pickups, named Profit-Maximizing Pickup and Delivery Selection Problem (PPDSP). Following the idea of searching through feasible routes, we propose a simple graph search that directly branches on feasible routes under pickup and delivery precedence requirements and uses depth-first search for the exact solution. The bounding of the search is provided by time windows, vehicle capacities and the potential gains in future routes. We then extended the graph search to the multi-vehicle case, in which we also developed a greedy heuristic for speedy solutions, and a Maximum Set Packing formulation for homogeneous and heterogeneous fleets.

Computational experiments showed that the graph search is able to solve single vehicle instances of up to 500 requests with tight time windows in seconds, and difficult instances of up to 400 requests with large time windows within minutes. Furthermore, the randomized search is able to find solutions with over $90 \%$ of the optimal profits in seconds for most instances. The extended graph search is able to optimally solve the multi-vehicle instances within an hour for up to 50 requests with tight time windows or up to 30 requests with large time windows. All solutions are faster than the benchmark solution of the Gurobi optimizer. The greedy heuristic finds routes with at least $86 \%$ optimal profits of up to 150 requests in seconds, and larger 
instances in minutes. Based on the reported performance of previous studies, we estimate that our solutions are sufficient to provide real-time decision support for carriers trading in freight exchanges, thus contributing to the improvement of the operational efficiency of the carriers and reducing the carbon footprint of road freight.

In future work, one could extend the experiments to more setups and integrate pre-processing of the requests, which, according to Arda et al. (2008), can greatly accelerate solution speed. We expect pre-processing to deliver tighter bounds and better traversing for the graph search. In addition, it is an intriguing idea to adapt column generation from Engineer et al. (2011); Ropke \& Cordeau (2009) to the current problem, even though this is likely to be non-trivial as our set-up embeds the resource-constrained shortest path problem inside a selection problem. Moreover, hybrid genetic algorithms with advanced diversity control can help effectively managing time windows (Vidal et al., 2013) or Finally, approaches from machine learning have received great traction lately as they can learn the inner structure of data and have proven themselves effective in several practical applications. Adapting such an approach to PPDSP would be an interesting path for future work.

\section{Acknowledgments}

We gratefully acknowledge the assistance of Franziska Brendle, Laura Cuthbertson and Ryan Grabowski.

\section{References}

Arda, Y., Crama, Y., \& Pironet, T. (2008). A Profitable Pickup and Delivery Problem with Time Windows. In 22nd Annual Conference of the Belgian Operations Research Society (ORBEL 22).

Baldacci, R., Bartolini, E., \& Mingozzi, A. (2011). An exact algorithm for the Pickup and Delivery Problem with Time Windows. Operations Research, 59, 414-426.

Berbeglia, G., Cordeau, J.-F., Gribkovskaia, I., \& Laporte, G. (2007). Static Pickup and Delivery Problems: A classification scheme and survey. TOP, 15, 1-31.

Caplice, C., \& Sheffi, Y. (2006). Combinatorial auctions for truckload transportation. In P. Cramton, Y. Shoham, \& R. Steinberg (Eds.), Combinatorial auctions (pp. 539572). Cambridge, MA: MIT Press.

Carrabs, F., Cerulli, R., \& Cordeau, J.-F. (2007). An additive branch-and-bound algorithm for the Pickup and Delivery Traveling Salesman Problem with LIFO or FIFO loading. Information Systems and Operational Research (INFOR), 45, 223-238.

Cherkesly, M., Desaulniers, G., \& Laporte, G. (2015). Branch-price-and-cut algorithms for the Pickup and Delivery Problem with Time Windows and LIFO loading. Transportation Science, 49, 752-766. 
Coja-Oghlan, A., Krumke, S. O., \& Nierhoff, T. (2006). A heuristic for the Stacker Crane Problem on trees which is almost surely exact. Journal of Algorithms, 61, $1-19$.

Cordeau, J.-F., \& Laporte, G. (2007). The dial-a-ride problem: models and algorithms. Annals of Operations Research, 153, 29-46.

Desrosiers, J., \& Lübbecke, M. E. (2005). A primer in column generation. In G. Desaulniers, J. Desrosiers, \& M. M. Solomon (Eds.), Column generation. New York, NJ: Springer.

Dumitrescu, I., Ropke, S., Cordeau, J.-F., \& Laporte, G. (2010). The Traveling Salesman Problem with Pickup and Delivery: Polyhedral results and a branch-and-cut algorithm. Mathematical Programming, 121, 269-305.

Engineer, F. G., Nemhauser, G. L., \& Savelsbergh, Martin W. P. (2011). Dynamic programming-based column generation on time-expanded networks: Application to the Dial-a-Flight Problem. INFORMS Journal on Computing, 23, 105-119.

Erdoğan, G., Cordeau, J.-F., \& Laporte, G. (2010). The Attractive Traveling Salesman Problem. European Journal of Operational Research, 203, 59-69.

Goel, A. (2010). A column generation heuristic for the General Vehicle Routing Problem. In C. Blum, \& R. Battiti (Eds.), Learning and Intelligent Optimization (pp. 1-9). Berlin and Heidelberg: Springer volume 6073 of Lecture Notes in Computer Science.

Goel, A., \& Gruhn, V. (2008). A General Vehicle Routing Problem. European Journal of Operational Research, 191, 650-660.

Gribkovskaia, I., Laporte, G., \& Shyshou, A. (2008). The Single Vehicle Routing Problem with Deliveries and Selective Pickups. Computers \& Operations Research, 35, 2908-2924.

Gurobi (2014). Gurobi Optimizer.

Gutiérrez-Jarpa, G., Desaulniers, G., Laporte, G., \& Marianov, V. (2010). A branchand-price algorithm for the Vehicle Routing Problem with Deliveries, Selective Pickups and Time Windows. European Journal of Operational Research, 206, 341-349.

Häme, L. (2011). An adaptive insertion algorithm for the single-vehicle Dial-a-Ride Problem with narrow time windows. European Journal of Operational Research, 209, 11-22.

Hernández-Pérez, H., \& Salazar-González, J.-J. (2007). The One-commodity Pickupand-Delivery Traveling Salesman Problem: Inequalities and algorithms. Networks, 50, 258-272.

Hernández-Pérez, H., \& Salazar-González, J.-J. (2009). The Multi-commodity oneto-one Pickup-and-Delivery Traveling Salesman Problem. European Journal of Operational Research, 196, 987-995.

Hernández-Pérez, H., \& Salazar-González, J.-J. (2014). The Multi-commodity Pickup- 
and-Delivery Traveling Salesman Problem. Networks, 63, 46-59.

Lee, H. L. (2004). The triple-A supply chain. Harvard Business Review, 82, 102-113.

Li, H., \& Lim, A. (2001). A metaheuristic for the pickup and delivery problem with time windows. In Tools with Artificial Intelligence, Proceedings of the 13th International Conference on (pp. 160-167).

Masson, R., Ropke, S., Lehuédé, F., \& Péton, O. (2014). A branch-and-cut-and-price approach for the Pickup and Delivery Problem with Shuttle routes. European Journal of Operational Research, 236, 849-862.

Mladenović, N., Urošević, D., Hanafi, S., \& Ilić, A. (2012). A general variable neighborhood search for the One-commodity Pickup-and-Delivery Travelling Salesman Problem. European Journal of Operational Research, 220, 270-285.

Parragh, S. N., Cordeau, J.-F., Doerner, K. F., \& Hartl, R. F. (2012). Models and algorithms for the heterogeneous Dial-a-Ride Problem with driver-related constraints. OR Spectrum, 34, 593-633.

Parragh, S. N., Doerner, K. F., \& Hartl, R. F. (2008). A survey on Pickup and Delivery Problems: Transportation between pickup and delivery locations: Part II. Journal für Betriebswirtschaft, 58, 81-117.

Rodríguez-Martín, I., \& Salazar-González, J. J. (2011). The Multi-Commodity Oneto-One Pickup-and-Delivery Traveling Salesman Problem: A matheuristic. Lecture Notes in Computer Science, 6701, 401-405.

Ropke, S., \& Cordeau, J.-F. (2009). Branch and cut and price for the Pickup and Delivery Problem with Time Windows. Transportation Science, 43, 267-286.

Schönberger, J., \& Kopfer, H. (2005). Planning the incorporation of logistics service providers to fulfill precedence- and time window-constrained transport requests in a most profitable way. In B. Fleischmann, \& A. Klose (Eds.), Distribution logistics (pp. 141-156). Berlin, New York: Springer volume 544 of Lecture Notes in Economics and Mathematical Systems.

Song, J., \& Regan, A. (2003). Combinatorial auctions for transportation service procurement: The carrier perspective. Transportation Research Record, 1833, 40-46.

Ting, C.-K., \& Liao, X.-L. (2013). The Selective Pickup and Delivery Problem: Formulation and a memetic algorithm. International Journal of Production Economics, 141, 199-211.

Toth, P., \& Vigo, D. (Eds.) (2002). The Vehicle Routing Problem. SIAM Monographs on Discrete Mathematics and Applications. Philadelphia, PA: Society for Industrial and Applied Mathematics.

Treleaven, K., Pavone, M., \& Frazzoli, E. (2013). Asymptotically optimal algorithms for one-to-one Pickup and Delivery pPoblems with applications to transportation systems. IEEE Transactions on Automatic Control, 58, 2261-2276. 
Vansteenwegen, P., Souffriau, W., \& van Oudheusden, D. (2011). The Orienteering Problem: A survey. European Journal of Operational Research, 209, 1-10.

Venkateshan, P., \& Mathur, K. (2011). An efficient column-generation-based algorithm for solving a Pickup-and-Delivery Problem. Computers \& Operations Research, 38, 1647-1655.

Vidal, T., Crainic, T. G., Gendreau, M., \& Prins, C. (2013). A hybrid genetic algorithm with adaptive diversity management for a large class of Vehicle Routing Problems with time-windows. Computers \& Operations Research, 40, 475-489. 